\title{
A survey of $\mathrm{HC}_{3} \mathrm{~N}$ in extragalactic sources
}

\section{Is $\mathrm{HC}_{3} \mathrm{~N}$ a tracer of activity in ULIRGs? ${ }^{\star}$}

\author{
J. E. Lindberg ${ }^{1,2,3, \star \star}$, S. Aalto ${ }^{3, \star \star \star}$, F. Costagliola ${ }^{3, \star \star \star \star}$, J.-P. Pérez-Beaupuits ${ }^{4,5}$, R. Monje ${ }^{6}$, and S. Muller ${ }^{3}$ \\ ${ }^{1}$ Centre for Star and Planet Formation, Natural History Museum of Denmark, University of Copenhagen, Øster Voldgade 5-7, \\ 1350 Copenhagen K, Denmark \\ e-mail: jlindberg@snm.ku.dk \\ 2 Nordic Optical Telescope, Apartado 474, 38700 Santa Cruz de La Palma, Santa Cruz de Tenerife, Spain \\ 3 Department of Earth and Space Sciences, Onsala Observatory, Chalmers University of Technology, 43992 Onsala, Sweden \\ 4 Max-Planck-Institut für Radioastronomie, Auf dem Hügel 69, 53121 Bonn, Germany \\ 5 Kapteyn Astronomical Institute, University of Groningen, Landleven 12, 9747 AD Groningen, The Netherlands \\ ${ }^{6}$ California Institute of Technology, 1200 E. California Blvd., Mail Code 301-17, Pasadena, CA 91125-4700, USA
}

Received 11 August 2010 / Accepted 16 December 2010

\begin{abstract}
Context. $\mathrm{HC}_{3} \mathrm{~N}$ is a molecule that is mainly associated with Galactic star-forming regions, but it has also been detected in extragalactic environments.

Aims. To present the first extragalactic survey of $\mathrm{HC}_{3} \mathrm{~N}$, when combining earlier data from the literature with six new single-dish detections, and to compare $\mathrm{HC}_{3} \mathrm{~N}$ with other molecular tracers $(\mathrm{HCN}, \mathrm{HNC}$ ), as well as other properties (silicate absorption strength, IR flux density ratios, $\mathrm{C}_{\text {II }}$ flux, and megamaser activity).

Methods. We present mm IRAM $30 \mathrm{~m}$, OSO $20 \mathrm{~m}$, and SEST observations of $\mathrm{HC}_{3} \mathrm{~N}$ rotational lines (mainly the $J=10-9$ transition) and of the $J=1-0$ transitions of $\mathrm{HCN}$ and $\mathrm{HNC}$. Our combined $\mathrm{HC}_{3} \mathrm{~N}$ data account for 13 galaxies (excluding the upper limits reported for the non-detections), while we have $\mathrm{HCN}$ and $\mathrm{HNC}$ data for more than 20 galaxies.

Results. A preliminary definition " $\mathrm{HC}_{3} \mathrm{~N}$-luminous galaxy" is made based upon the $\mathrm{HC}_{3} \mathrm{~N} / \mathrm{HCN}$ ratio. Most $(\sim 80 \%)$ HC 3 N-luminous galaxies seem to be deeply obscured galaxies and (U)LIRGs. A majority ( $\sim 60 \%$ or more) of the $\mathrm{HC}_{3} \mathrm{~N}$-luminous galaxies in the sample present $\mathrm{OH}$ mega- or strong kilomaser activity. A possible explanation is that both $\mathrm{HC}_{3} \mathrm{~N}$ and $\mathrm{OH}$ megamasers need warm dust for their excitation. Alternatively, the dust that excites the $\mathrm{OH}$ megamaser offers protection against $\mathrm{UV}$ destruction of $\mathrm{HC}_{3} \mathrm{~N}$. $\mathrm{A}$ high silicate absorption strength is also found in several of the $\mathrm{HC}_{3} \mathrm{~N}$-luminous objects, which may help the $\mathrm{HC}_{3} \mathrm{~N}$ to survive. Finally, we find that a high $\mathrm{HC}_{3} \mathrm{~N} / \mathrm{HCN}$ ratio is related to a high dust temperature and a low $\mathrm{C}_{\text {II }}$ flux.
\end{abstract}

Key words. galaxies: ISM - galaxies: starburst - galaxies: active - radio lines: galaxies - radio lines: ISM - ISM: molecules

\section{Introduction}

Finding useful tracers of the interaction between the activity in galaxy nuclei and surrounding interstellar medium (ISM) is an important and growing aspect of current extragalactic molecular astronomy. In this context, single dish surveys of polar molecules such as $\mathrm{HCN}, \mathrm{HNC}, \mathrm{HCO}^{+}$, and $\mathrm{CS}$ have been used to investigate possible correlations between molecular line ratios and type/intensity of activity (e.g. Kohno et al. 2001; Aalto et al. 2002; Imanishi et al. 2004; Graciá-Carpio et al. 2006; Krips et al. 2008; Baan et al. 2008). For example, it has been suggested that an elevated $\mathrm{HCN} / \mathrm{HCO}^{+} 1-0$ line intensity ratio indicates the presence of an AGN (Graciá-Carpio et al. 2006). Around an active galactic nucleus (AGN) the chemistry is supposedly dominated by hard X-rays in an X-ray dominated region (XDR),

\footnotetext{
* Appendix is only available in electronic form at http://www . aanda.org

$\star \star$ J.L. wishes to thank Instrumentcenter for Danish Astrophysics (IDA) for grant support.

$\star \star \star$ S.A. wishes to thank the Swedish Research Council for grant support.

$\star \star \star \star$ F.C. wishes to thank the EU ESTRELA programme for support.
}

and some chemical models predict an abundance enhancement of $\mathrm{HCN}$ paired with selective destruction of $\mathrm{HCO}^{+}$(Maloney et al. 1996) - which could lead to an elevated $\mathrm{HCN} / \mathrm{HCO}^{+}$line ratio (under the circumstances that the line ratio directly reflects the abundance ratio). However, more recent chemical models instead suggest that $\mathrm{HCO}^{+}$is enhanced in XDRs (Meijerink \& Spaans 2005; Meijerink et al. 2007), and $\mathrm{HCO}^{+}$is also expected to be under-abundant in regions of very young star formation (Aalto 2008), so the line ratio is ambiguous.

Other molecular tracers could help resolve the dichotomy of the $\mathrm{HCN} / \mathrm{HCO}^{+}$line ratio. The serendipitous discovery of the $J=10-9$ line of $\mathrm{HC}_{3} \mathrm{~N}$ near the HNC $J=1-0$ line in a survey by Aalto et al. (2002) led us to look more closely at this molecule. $\mathrm{HC}_{3} \mathrm{~N}$ is the simplest of the cyanopolyynes (carbon chains with an attached $\mathrm{CN}$ group) and is a grain chemistry product, in contrast to molecules such as $\mathrm{HCN}$ and $\mathrm{HCO}^{+} . \mathrm{HC}_{3} \mathrm{~N}$ thrives in warm, dense shielded regions such as hot cores where abundances can reach $10^{-8}$ or even higher, since it is easily destroyed by photo-dissociation (Rodriguez-Franco et al. 1998) and $\mathrm{C}^{+}$ions (Prasad \& Huntress 1980). Therefore, $\mathrm{HC}_{3} \mathrm{~N}$ line emission could be used to identify galaxies where star formation is in the early, embedded stage of its evolution. Recently, $\mathrm{HC}_{3} \mathrm{~N}$ 
was found in high abundance in the highly obscured galaxy NGC 4418 (Aalto et al. 2007), as well as the ULIRG Arp 220 (Aalto et al. 2002).

We have searched for $\mathrm{HC}_{3} \mathrm{~N}$ line emission in a sample of galaxies in various stages and types of activity: AGNs, starbursts, and ultraluminous galaxies (ULIRGs). In some of the galaxies the nature of the activity is elusive since it is embedded in huge columns of dust absorbing emission at optical and infrared wavelengths. In some cases, the extinction is so strong that no emission emerges at optical or IR wavelengths requiring us to probe the nature of the activity at radio and $\mathrm{mm}$ wavelengths. $\mathrm{HC}_{3} \mathrm{~N}$ has a rich $\mathrm{mm}$ and sub-mm wavelength spectrum consisting of a multitude of rotational and vibrational lines often appearing close to each other in the same band. Through its vibrational transitions, $\mathrm{HC}_{3} \mathrm{~N}$ responds strongly to the IR field from dusty nuclei (Costagliola \& Aalto 2010). Therefore, combining the rotational and vibrational line information of $\mathrm{HC}_{3} \mathrm{~N}$ allows us to study the abundance of $\mathrm{HC}_{3} \mathrm{~N}$ (comparing with chemical models of XDRs and starbursts) as well as the intensity and temperature structure of the buried IR source.

Rotational lines of vibrationally excited $\mathrm{HC}_{3} \mathrm{~N}$ have recently been discovered in a few galaxies (NGC 4418 (Costagliola \& Aalto 2010), Arp 220 (Martín et al. 2011), and IC 860 (Costagliola et al. 2011)), therefore showing that it is important to take both radiative and collisional excitation into consideration when interpreting $\mathrm{HC}_{3} \mathrm{~N}$ line emission from IR luminous galaxies.

It can also be noted that absorption lines of $\mathrm{HC}_{3} \mathrm{~N}$ has been found in a $z \sim 0.89$ galaxy located in front of the quasar PKS 1830-211 (Henkel et al. 2009).

\subsection{Outline}

Here, the first survey of extragalactic $\mathrm{HC}_{3} \mathrm{~N}$ data is presented. We report new $\mathrm{HC}_{3} \mathrm{~N}$ observations in 19 galaxies (detections in six of them), mainly (U)LIRGs and starburst galaxies, and complete this sample with data from all earlier extragalactic $\mathrm{HC}_{3} \mathrm{~N}$ emission line single-dish detections found in the literature. The aim of the study is to compare the $\mathrm{HC}_{3} \mathrm{~N}$ luminosity with other molecular tracers as well as galaxy properties to see if the presence of $\mathrm{HC}_{3} \mathrm{~N}$ can be used to predict other galaxy properties, e.g. the source of activity in the galaxy.

In Sect. 2, the general properties of $\mathrm{HC}_{3} \mathrm{~N}$ in space are discussed. In Sect. 3 we present the new observations and discuss the collection of data from the literature. In Sect. 4 we present the results in terms of line intensities and line ratios. In Sect. 5 we discuss the interpretation of the $\mathrm{HC}_{3} \mathrm{~N}$ results and compare them with silicate absorption strength (Sect. 5.2), OH megamaser activity (Sect. 5.3), IR flux density ratios (Sect. 5.4), C II flux (Sect. 5.5), and the HNC/HCN 1-0 line ratio (Sect. 5.6). In Sect. 5.7 future studies resulting from this project are discussed.

\section{2. $\mathrm{HC}_{3} \mathrm{~N}$ in space}

\subsection{Generation of $\mathrm{HC}_{3} \mathrm{~N}$}

Acetylene, $\mathrm{C}_{2} \mathrm{H}_{2}$, exists on grains in the ISM (Chapman et al. 2009). After evaporating from the grains there are at least two different paths the $\mathrm{C}_{2} \mathrm{H}_{2}$ may follow. If a high UV field is present (the region being a PDR), it will photo-dissociate into the ethynyl radical, $\mathrm{C}_{2} \mathrm{H}$ (Meier \& Turner 2005; Cherchneff et al. 1993; Heikkilä et al. 1999):

$$
\mathrm{C}_{2} \mathrm{H}_{2}+h v \longrightarrow \mathrm{C}_{2} \mathrm{H}+\mathrm{H} .
$$

If no strong UV field is present (no PDR), but $\mathrm{CN}$ (the cyano radical) is available for reactions, the $\mathrm{C}_{2} \mathrm{H}_{2}$ will instead react with the $\mathrm{CN}$ to form $\mathrm{HC}_{3} \mathrm{~N}$ (Meier \& Turner 2005; Chapman et al. 2009; Fukuzawa \& Osamura 1997):

$$
\mathrm{C}_{2} \mathrm{H}_{2}+\mathrm{CN} \longrightarrow \mathrm{HC}_{3} \mathrm{~N}+\mathrm{H} \text {. }
$$

This hypothesis is strengthened by interferometric maps of $\mathrm{HC}_{3} \mathrm{~N}$ and $\mathrm{C}_{2} \mathrm{H}$ in IC 342 found in Meier \& Turner (2005). The maps show a clear anti-correlation between the distributions of the molecules. Using the abundances of $\mathrm{HC}_{3} \mathrm{~N}, \mathrm{C}_{2} \mathrm{H}$, and $\mathrm{CN}$ in a region where it is expected that grains with $\mathrm{C}_{2} \mathrm{H}_{2}$ first were present, it should therefore be possible to tell whether a strong UV field is present or not.

Irvine et al. (1987) find that $\mathrm{C}_{2} \mathrm{H}$, contrarily to $\mathrm{HC}_{3} \mathrm{~N}$, is two orders of magnitude more abundant in the Orion ridge than in its hot core. Possibly, the hot core shields the $\mathrm{HC}_{3} \mathrm{~N}$ and $\mathrm{C}_{2} \mathrm{H}_{2}$ from photo-dissociating into $\mathrm{C}_{2} \mathrm{H}$. Some of the $\mathrm{C}_{2} \mathrm{H}_{2}$ instead reacts with the $\mathrm{CN}$ (although not very abundant) and forms even more $\mathrm{HC}_{3} \mathrm{~N}$. The highest $\mathrm{HC}_{3} \mathrm{~N}$ abundances are found in Sgr B2 hot cores, being in the order of $10^{-7}$ of the $\mathrm{H}_{2}$ abundance (de Vicente et al. 2000).

We have not included all possible $\mathrm{HC}_{3} \mathrm{~N}$ formation mechanisms here, and investigations of other processes are ongoing, as for example the notion of ice formation of $\mathrm{HC}_{3} \mathrm{~N}$ (Szczepanski et al. 2005).

\subsection{Destruction of $\mathrm{HC}_{3} \mathrm{~N}$}

In the Galaxy, $\mathrm{HC}_{3} \mathrm{~N}$ is associated with warm, dense, shielded gas around young stars or star-forming regions, and is easily destroyed by UV radiation and reactions with $\mathrm{C}^{+}$ions (RodriguezFranco et al. 1998; Meier \& Turner 2005). It will form either $\mathrm{C}_{2} \mathrm{H}$ or $\mathrm{C}_{3} \mathrm{~N}$ when being photo-dissociated (Cherchneff et al. 1993), and $\mathrm{C}_{3} \mathrm{H}^{+}$or $\mathrm{C}_{4} \mathrm{~N}^{+}$when reacting with $\mathrm{C}^{+}$ (Bohme \& Raksit 1985). Among the possible reactions destroying $\mathrm{HC}_{3} \mathrm{~N}$ are:

$$
\begin{aligned}
& \mathrm{HC}_{3} \mathrm{~N}+h v \longrightarrow \mathrm{C}_{2} \mathrm{H}+\mathrm{CN} \\
& \mathrm{HC}_{3} \mathrm{~N}+h v \longrightarrow \mathrm{C}_{3} \mathrm{~N}+\mathrm{H} \\
& \mathrm{HC}_{3} \mathrm{~N}+\mathrm{C}^{+} \longrightarrow \mathrm{C}_{3} \mathrm{H}^{+}+\mathrm{CN} \\
& \mathrm{HC}_{3} \mathrm{~N}+\mathrm{C}^{+} \longrightarrow \mathrm{C}_{4} \mathrm{~N}^{+}+\mathrm{H}
\end{aligned}
$$

Reaction rates of these reactions, as well as those in Sect. 2.1, can be found in e.g. Cherchneff et al. (1993).

\subsection{Abundances of $\mathrm{HC}_{3} \mathrm{~N}$}

Irvine et al. (1987) give the relative abundances of several molecules in the core and ridge of the Orion molecular cloud. The detections of these molecules in the Galaxy are indicative of their abundances in high and low density molecular regions. The relative $\mathrm{HC}_{3} \mathrm{~N}$ abundance lies around $10^{-9}$ of the $\mathrm{H}_{2}$ abundance in the core (dense region), and $10^{-10}$ in the ridge (low density region). This is a relatively small difference between high and low density regions, as compared to e.g. HCN, with about $10^{-7}$ of the $\mathrm{H}_{2}$ abundance in the core, and $10^{-9}$ in the ridge.

The intense radiation from starburst regions and/or AGNs in the centre of many galaxies will turn surrounding gas clouds into regions where the chemical structure depends highly on the radiation field, either photon-dominated regions (PDRs) or X-ray dominated regions (XDRs). In XDRs the abundance of several molecules (e.g. $\mathrm{CN}$ and $\mathrm{CH}_{2}$ ) are expected to be enhanced with respect to the abundance commonly found in PDRs. Due to the 
Table 1. Data for the observations reported in this work.

\begin{tabular}{|c|c|c|c|c|}
\hline Galaxy & Molecule & Line & Telescope & Date \\
\hline Arp 220 & $\mathrm{HC}_{3} \mathrm{~N}$ & $10-9$ & OSO & $2001-11-11$ \\
\hline Arp 220 & $\mathrm{HC}_{3} \mathrm{~N}$ & $12-11$ & OSO & 2001-11-09 \\
\hline Circinus & HNC & $1-0$ & SEST & $2001-01-14$ \\
\hline IC 694 & $\mathrm{HC}_{3} \mathrm{~N}$ & $12-11$ & OSO & 2001-11-10 \\
\hline IC 860 & $\mathrm{HC}_{3} \mathrm{~N}$ & $28-27$ & IRAM & $2007-12-16$ \\
\hline IC 860 & HNC & $1-0$ & IRAM & $2007-12-16$ \\
\hline I17208 & $\mathrm{HC}_{3} \mathrm{~N}$ & $10-9$ & IRAM & $2007-12-16$ \\
\hline I17208 & HNC & $1-0$ & IRAM & 2006-06-28 \\
\hline Maffei 2 & $\mathrm{HC}_{3} \mathrm{~N}$ & $12-11$ & IRAM & $2007-08-25$ \\
\hline NGC 34 & HNC & $1-0$ & SEST & 2001-01-14 \\
\hline NGC 613 & $\mathrm{HCN}$ & $1-0$ & SEST & 2001-02-11 \\
\hline NGC 613 & HNC & $1-0$ & SEST & 2001-02-13 \\
\hline NGC 1056 & $\mathrm{HC}_{3} \mathrm{~N}$ & $16-15$ & IRAM & $2006-06-30$ \\
\hline NGC 1056 & HNC & $1-0$ & IRAM & $2006-06-30$ \\
\hline NGC 1377 & $\mathrm{HC}_{3} \mathrm{~N}$ & $16-15$ & IRAM & $2007-12-13$ \\
\hline NGC 1377 & $\mathrm{HC}_{3} \mathrm{~N}$ & $25-24$ & IRAM & $2007-12-13$ \\
\hline NGC 1377 & $\mathrm{HCN}$ & $1-0$ & IRAM & $2007-12-13$ \\
\hline NGC 1377 & HNC & $1-0$ & IRAM & $2007-12-13$ \\
\hline NGC 1614 & HNC & $1-0$ & SEST & 2001-02-13 \\
\hline NGC 2146 & $\mathrm{HC}_{3} \mathrm{~N}$ & $10-9$ & OSO & 2001-11-08 \\
\hline NGC 2146 & $\mathrm{HC}_{3} \mathrm{~N}$ & $12-11$ & OSO & 2001-11-08 \\
\hline NGC 2623 & $\mathrm{HC}_{3} \mathrm{~N}$ & $12-11$ & OSO & $2001-11-12$ \\
\hline NGC 3079 & $\mathrm{HC}_{3} \mathrm{~N}$ & $10-9$ & IRAM & 2006-05-14 \\
\hline NGC 3079 & $\mathrm{HC}_{3} \mathrm{~N}$ & $16-15$ & IRAM & 2006-05-14 \\
\hline NGC 3079 & $\mathrm{HC}_{3} \mathrm{~N}$ & $25-24$ & IRAM & 2006-05-14 \\
\hline NGC 3690 & $\mathrm{HC}_{3} \mathrm{~N}$ & $12-11$ & OSO & 2001-11-09 \\
\hline NGC 4418 & $\mathrm{HCN}$ & $1-0$ & IRAM & 2008-07-19 \\
\hline NGC 4945 & $\mathrm{HNC}$ & $1-0$ & SEST & 2001-01-15 \\
\hline NGC 5135 & HNC & $1-0$ & SEST & 2001-01-13 \\
\hline NGC 6946 & $\mathrm{HC}_{3} \mathrm{~N}$ & $12-11$ & IRAM & $2007-08-25$ \\
\hline UGC 5101 & $\mathrm{HC}_{3} \mathrm{~N}$ & $10-9$ & IRAM & $2007-12-13$ \\
\hline UGC 5101 & HNC & $1-0$ & IRAM & 2007-12-13 \\
\hline
\end{tabular}

Notes. For the observations cited from the literature, see the respective articles referred to in Tables 4-6. We would like to point out that some of the $\mathrm{HC}_{3} \mathrm{~N}$ 10-9 data reported by us come from SEST HNC 1-0 spectra.

high $\mathrm{C}^{+}$abundance in XDRs, a low $\mathrm{HC}_{3} \mathrm{~N}$ abundance is expected (Aalto 2008). The $\mathrm{HC}_{3} \mathrm{~N} / \mathrm{CN}$ abundance ratio found in PDRs is also very low compared to the same ratio measured in hot, dense cores (Rodriguez-Franco et al. 1998). For further discussion about PDR and XDR chemistry, see e.g. Tielens \& Hollenbach (1985); Lepp \& Dalgarno (1996); Meijerink \& Spaans (2005); Maloney et al. (1996).

\section{Observations}

The new observations reported in this work were carried out with the IRAM $30 \mathrm{~m}^{1}$, OSO $20 \mathrm{~m}$, and SEST $15 \mathrm{~m}$ telescopes between 2001 and 2008. Detailed lists with system temperatures and dates for the observations reported in this work are found in Table 1). The pointing accuracy was better than $2^{\prime \prime}$ for all the observations, and typical system temperatures were $150 \mathrm{~K}$ (IRAM 90-110 GHz), $250 \mathrm{~K}$ (IRAM $125 \mathrm{GHz}$ ), $400 \mathrm{~K}$ (IRAM 225-250 GHz), and $300 \mathrm{~K}$ (OSO and SEST). In this survey we also include data from the literature using the already mentioned telescopes, as well as the NRO $45 \mathrm{~m}$, NRAO $12 \mathrm{~m}$, and FCRAO 14 m telescopes. Whenever using data from the literature, the beam sizes and efficiencies given in the respective

\footnotetext{
1 Based on observations carried out with the IRAM 30m Telescope. IRAM is supported by INSU/CNRS (France), MPG (Germany), and IGN (Spain).
}

Table 2. Observational parameters.

\begin{tabular}{|c|c|c|c|}
\hline Transition & $v[\mathrm{GHz}]^{a}$ & $H P B W\left[{ }^{\prime \prime}\right]^{b}$ & $\eta_{\mathrm{mb}}^{b}$ \\
\hline \multicolumn{4}{|l|}{ IRAM: } \\
\hline $\mathrm{HC}_{3} \mathrm{~N} 10-9$ & 90.979 & 28 & 0.80 \\
\hline $\mathrm{HC}_{3} \mathrm{~N} 12-11$ & 109.174 & 24 & 0.73 \\
\hline $\mathrm{HC}_{3} \mathrm{~N} 16-15$ & 145.561 & 17 & 0.67 \\
\hline $\mathrm{HC}_{3} \mathrm{~N} 25-24$ & 227.419 & 10.5 & 0.63 \\
\hline $\mathrm{HC}_{3} \mathrm{~N} 28-27$ & 254.699 & 9 & 0.59 \\
\hline $\mathrm{HCN} 1-0$ & 88.632 & 28 & 0.80 \\
\hline HNC $1-0$ & 90.664 & 28 & 0.80 \\
\hline \multicolumn{4}{|l|}{ OSO: } \\
\hline $\mathrm{HC}_{3} \mathrm{~N} 12-11$ & 109.174 & 36 & 0.52 \\
\hline $\mathrm{HC}_{3} \mathrm{~N} 10-9$ & 90.979 & 42 & 0.59 \\
\hline \multicolumn{4}{|l|}{ SEST: } \\
\hline $\mathrm{HC}_{3} \mathrm{~N} 10-9$ & 90.979 & 55 & 0.75 \\
\hline $\mathrm{HCN} 1-0$ & 88.632 & 57 & 0.75 \\
\hline HNC $1-0$ & 90.664 & 55 & 0.75 \\
\hline
\end{tabular}

Notes. ${ }^{(a)}$ From the NIST database Recommended Rest Frequencies for Observed Interstellar Molecular Microwave Transitions (http:// physics.nist.gov/cgi-bin/micro/table5/start.pl).

(b) The half-power beamwidths and main beam efficiencies are collected from the respective telescope web pages.

articles have been used for calculations of line ratios. The parameters used for all new observations reported in this work are given in Table 2.

One could argue that the many different instruments used to obtain the data in this article might introduce a bias difficult to compensate for. However, when comparing the HNC/HCN 1-0 line ratios obtained with IRAM and SEST respectively, no systematic bias is detected. The average line ratio was calculated to $0.51 \pm 0.11$ for SEST data and $0.51 \pm 0.08$ for IRAM data.

All objects investigated (observed by us or with data from the literature) are listed in Table 3, along with some important characteristics.

The relative $\mathrm{HC}_{3} \mathrm{~N}$ abundances calculated in this work will be expressed as line ratios between an $\mathrm{HC}_{3} \mathrm{~N}$ line (mostly the $J=$ 10-9 transition) and the $J=1-0$ transitions of $\mathrm{HCN}$ and HNC. These two molecules are chosen as they are good tracers of high density regions (see e.g. Papadopoulos 2007; Aalto et al. 2002), where we expect to find the $\mathrm{HC}_{3} \mathrm{~N}$ (Meier \& Turner 2005). Also, $\mathrm{HCN}$ and $\mathrm{HNC}$ data are available for most of the objects in the sample. We note that the line ratios are not linearly proportional to ratios between the abundances of the species, since they will also depend on excitation conditions and optical depths in the galaxies. A high $\mathrm{HC}_{3} \mathrm{~N} / \mathrm{HCN}$ ratio might thus sometimes be a tracer of discrepancies in temperatures, densities, or IR pumping in the galaxies.

A discussion of the method used when calculating the line ratios can be found in Appendix A. A few of the most nearby galaxies in the survey have such a large angular distribution that the measured values might not represent a global value for molecular gas in the galaxy, but rather a value for a certain (central) region of the galaxy. This effect is discussed in Appendix A.1.

The sample of galaxies observed by us has been chosen to have a high probability of finding $\mathrm{HC}_{3} \mathrm{~N}-$ it is by no means intended to be an unbiased sample of some random galaxies, and thus the relatively high detection ratio should definitely not reflect the amount of $\mathrm{HC}_{3} \mathrm{~N}$-luminous galaxies in the universe. The same is most likely true for the galaxies found in the literature. Another important selection effect for the objects from the 
Table 3. List of investigated objects and some of their properties. For the galaxies with new observations reported, the given positions are those used for our observations. For objects not observed by us the position in NED (2009) is given.

\begin{tabular}{|c|c|c|c|c|c|c|c|}
\hline Galaxy & $\begin{array}{l}\text { RA } \\
(\mathrm{J} 2000.0)\end{array}$ & $\begin{array}{l}\text { Dec } \\
(\mathrm{J} 2000.0)\end{array}$ & Type $^{a}$ & $\begin{array}{c}c z^{b} \\
{\left[\mathrm{~km} \mathrm{~s}^{-1}\right]}\end{array}$ & $\begin{array}{r}D^{c} \\
{[\mathrm{Mpc}]}\end{array}$ & $\begin{array}{r}\log L_{\mathrm{FIR}}{ }^{d} \\
{\left[L_{\odot}\right]}\end{array}$ & $\begin{array}{c}\theta_{\mathrm{HCN}}^{e} \\
{\left[{ }^{\prime \prime}\right]}\end{array}$ \\
\hline Arp 220 & 153457.1 & +233011.3 & ULIRG, Obsc., SB? & 5450 & 78.1 & 12.15 & $2^{1}$ \\
\hline Circinus & 141034.3 & -645212.5 & AGN, cp? & $434^{f}$ & 3.13 & & $21(\mathrm{CO} 3-2)^{2}$ \\
\hline IC 342 & 034648.5 & +680546 & SB & 31 & 4.00 & 10.01 & $20^{3}$ \\
\hline IC $694^{g}$ & 112833.6 & +583346.0 & SB & 3159 & 58.2 & $11.74^{h}$ & $5^{4}$ \\
\hline IC 860 & 131503.5 & +243708.0 & Obsc. & 3887 & $59.1^{i}$ & 11.14 & $*$ \\
\hline $\mathrm{I} 17208^{j}$ & 172321.9 & -001700.9 & ULIRG, Obsc., SB? & 12852 & 178 & 12.35 & $0.67^{5}$ \\
\hline M82 & 095552.7 & +694046 & SB & 187 & 5.68 & 10.61 & $>30^{1}$ \\
\hline Maffei 2 & 024155.1 & +593615.0 & SB & $-17^{f}$ & 3.34 & & $20 \times 7^{6}$ \\
\hline NGC 34 & 001106.5 & -120626.6 & SB & 5931 & 79.8 & 11.34 & * \\
\hline NGC 253 & 004733.1 & -251718 & SB & 261 & 3.22 & 10.29 & $18 \times 8^{7}$ \\
\hline NGC 613 & 013636.7 & -290950.4 & $\mathrm{cp}$ & 1475 & 18.6 & 10.22 & $40(\operatorname{CO} 1-0)^{8}$ \\
\hline NGC 1056 & 024248.3 & +283427.1 & $\mathrm{AGN}$ & 1545 & 22.7 & 9.79 & 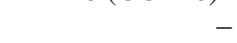 \\
\hline NGC 1068 & 024240.7 & -000047.0 & $\mathrm{cp}$ & 1005 & 15.3 & 10.89 & $4.5^{1}$ \\
\hline NGC 1365 & 033336.4 & -360826.1 & $\mathrm{cp}$ & 1636 & 19.9 & 10.86 & $34(\mathrm{CO} 2-1)^{9}$ \\
\hline NGC 1377 & 033639.1 & -205408.0 & Obsc., AGN? & 1792 & 22.5 & 9.95 & - \\
\hline NGC 1614 & 043624.2 & -082840.3 & SB & 4746 & 63.4 & 11.43 & $12(\mathrm{CO} 2-1)^{10}$ \\
\hline NGC 1808 & 050742.3 & -373047 & $\mathrm{SB}, \mathrm{cp}$ ? & 1000 & 11.2 & 10.55 & $18(\mathrm{CO} 2-1)^{10}$ \\
\hline NGC 2146 & 061837.8 & +782122.9 & SB & 885 & 16.9 & 10.93 & $20^{1}$ \\
\hline NGC 2623 & 083824.1 & +254517.2 & SB & 5538 & 76.9 & 11.48 & $1.8^{11}$ \\
\hline NGC 3079 & 100157.81 & +554047.1 & SB? AGN? & 1142 & 19.7 & 10.65 & $13 \times 5(\text { CO } 1-0)^{12}$ \\
\hline NGC 3256 & 102751.3 & -435414 & SB & 2781 & 36.5 & 11.43 & $9(\mathrm{CO} 2-1)^{10}$ \\
\hline NGC $3690^{g}$ & 112831.0 & +583340.0 & SB & 3159 & 46.9 & $11.74^{h}$ & $1.56^{5}$ \\
\hline NGC 4418 & $122654.8^{k}$ & $-005242.0^{k}$ & Obsc., AGN? & 2104 & 32.6 & 11.00 & $5(\mathrm{CO} 1-0)^{13}$ \\
\hline NGC 4945 & 130527.0 & -492804.5 & $\mathrm{SB}, \mathrm{cp} ?$ & 560 & 4.85 & 10.41 & $15(\mathrm{CO} 3-2)^{2}$ \\
\hline NGC 5135 & 132544.0 & -295002.2 & $\mathrm{cp}$ & 4114 & 56.0 & 11.06 & $15 \times 5(\mathrm{CO} 1-0)^{14}$ \\
\hline NGC 6946 & 203452.3 & +600914.0 & SB & 53 & 5.64 & 10.01 & $10^{1}$ \\
\hline NGC 7130 & 214819.5 & -345705 & $\mathrm{cp}$ & 4824 & 65.4 & 11.23 & $10(\mathrm{CO} 1-0)^{10}$ \\
\hline UGC 5101 & 093551.6 & +612111.7 & LIRG, $\mathrm{cp}$ & 11785 & 165 & 11.87 & $3.50(\mathrm{CO} 1-0)^{5}$ \\
\hline
\end{tabular}

References. (1) Table in Krips et al. (2008); (2) Table in Curran et al. (2001a); (3) HCN map in Meier \& Turner (2005); (4) HCN map in Aalto et al. (1997); (5) Table in Graciá-Carpio et al. (2008); (6) HCN map in Nguyen-Rieu et al. (1994); (7) HCN map in Knudsen et al. (2007); (8) CO 1-0 map in Bajaja et al. (1995); (9) CO 2-1 source size in Curran et al. (2001b); (10) Table in Aalto et al. (1995); (11) Table in Bryant \& Scoville (1999); (12) CO 1-0 map in Koda et al. (2002); (13) CO 1-0 map in Dale et al. (2005); (14) CO 1-0 map in Regan et al. (1999).

Notes. ${ }^{(a)}$ The classifications have been obtained by careful investigation of the notes in NED (2009). SB = starburst, AGN = active galactic nucleus, $\mathrm{cp}=$ composite of SB and AGN, Obsc. = obscured, ULIRG = Ultra-luminous Infrared galaxy, LIRG = Luminous Infrared galaxy. ${ }^{(b)}$ Heliocentric radial velocity of source, from Sanders et al. (2003). ${ }^{(c)}$ Distance to source, corrected for Virgo infall only, from NED (2009). (d) Far Infrared Luminosity, from Sanders et al. (2003). ${ }^{(e)}$ Source sizes, given for HCN 1-0 line if not specified otherwise. For galaxies with an asterisk (*), no value has been found, but $D \gtrsim 45 \mathrm{Mpc}$, allowing the point-like approximation $\left(\theta_{\mathrm{HCN}}=0\right)$ with an error $\lesssim 5 \%$ if the dense molecular gas in this galaxy is not unusually widely distributed. For galaxies marked with a dash (-), no value has been found, and $D<45 \mathrm{Mpc}{ }^{(f)}$ From NED (2009). ${ }^{(g)}$ IC 694 and NGC 3690 are together also known as the merger Arp 299. ${ }^{(h)}$ This is the FIR luminosity of IC 694 and NGC 3690 together. ${ }^{(i)}$ From Sanders et al. (2003). ${ }^{(j)}$ Short for IRAS 17208-0014. ${ }^{(k)}$ For the HCN 1-0 data the following coordinates have been used: RA 12 26 54.63, Dec -00 5239.6 (J2000.0).

literature is that detections are much more likely to be reported than non-detections, which also leads to a biased sample.

To increase the chance of detecting $\mathrm{HC}_{3} \mathrm{~N}$, almost all of the galaxies that were chosen to be part of the sample have earlier detections of $\mathrm{HCN}$, which means that they should have large amounts of dense gas, increasing the possibility of finding $\mathrm{HC}_{3} \mathrm{~N}$. As the goal of the study is to investigate if $\mathrm{HC}_{3} \mathrm{~N}$ can trace the source of the activity in active galaxies, the sample consists only of active galaxies - starburst galaxies and AGN galaxies (the source of the activity is although disputed or unknown in many of the galaxies in the sample).

Throughout the article, the $T_{\mathrm{A}}^{*}$ scale will be used for all our data. For the data from the literature, the temperature scale used in each article will be used in our tables, clearly noted whenever the $T_{\mathrm{A}}^{*}$ scale is not used. This will make it easier to detect any errors that might have occurred in the survey work. When the line ratios are calculated, the efficiencies will be taken into account properly.
Data analysis was performed with the X-Spec ${ }^{2}$ software package. A first order baseline was subtracted from all spectra.

\section{Results}

All new $\mathrm{HC}_{3} \mathrm{~N}, \mathrm{HCN}$, and $\mathrm{HNC}$ spectra reported in this work are displayed in Figs. 1-7. The observed values of the spectral line intensities can be found in Tables 4-6. Data from the literature are also included in these tables.

Some observations of $\mathrm{HC}_{3} \mathrm{~N} 10-9$ and $\mathrm{HNC}$ 1-0 performed with SEST include both these lines in the same spectrum, due to the large bandwidth. The spectra are in these cases labelled according to the central peak. The frequency difference between the two peaks is $315 \mathrm{MHz}$, as can be seen in Table 2. This gives a velocity difference of approximately $1000 \mathrm{~km} \mathrm{~s}^{-1}$.

\footnotetext{
2 http://www. chalmers.se/rss/oso-en/observations/ data-reduction-software
} 
Table 4. Data from $\mathrm{HC}_{3} \mathrm{~N}$ observations.

\begin{tabular}{|c|c|c|c|c|c|c|c|c|c|}
\hline Galaxy & Line & $\begin{array}{l}I\left(\mathrm{HC}_{3} \mathrm{~N}\right)^{a} \\
{\left[\mathrm{~K} \mathrm{~km} \mathrm{~s}^{-1}\right]}\end{array}$ & $\begin{array}{c}S_{v} \Delta v \\
{\left[\mathrm{Jy} \mathrm{km} \mathrm{s}^{-1} \text { ] }\right.}\end{array}$ & $\begin{array}{c}\Delta v \\
{\left[\mathrm{~km} \mathrm{~s}^{-1}\right]}\end{array}$ & Telescope & $\eta_{\mathrm{mb}}$ & $\begin{array}{l}\theta_{\mathrm{mb}} \\
{\left[{ }^{\prime \prime}\right]}\end{array}$ & $T$ scale $^{a}$ & References \\
\hline Arp 220 & $10-9$ & $2.02 \pm 0.15$ & $45 \pm 3$ & 340 & OSO $20 \mathrm{~m}$ & 0.59 & 44 & $T_{\mathrm{A}}^{*}$ & (1) \\
\hline Arp 220 & $10-9^{b}$ & $0.4 \pm 0.15$ & $11 \pm 4$ & 350 & SEST $15 \mathrm{~m}$ & 0.75 & 55 & $T_{\mathrm{A}}^{*}$ & (2) \\
\hline Arp 220 & $12-11$ & $0.96 \pm 0.1$ & $23 \pm 2$ & 170 & OSO $20 \mathrm{~m}$ & 0.52 & 36 & $T_{\mathrm{A}}^{*}$ & (1) \\
\hline Circinus & $10-9^{b}$ & $1.01 \pm 0.1$ & $27.6 \pm 2.7$ & 290 & SEST $15 \mathrm{~m}$ & 0.75 & 55 & $T_{\mathrm{A}}^{*}$ & (1) \\
\hline IC 342 & $10-9$ & $2.6 \pm 0.7$ & $14 \pm 4$ & 52 & IRAM $30 \mathrm{~m}$ & 0.8 & 25 & $T_{\mathrm{R}}^{*}$ & (3) \\
\hline IC 694 & $12-11$ & $<0.30$ & $<7.4$ & . & OSO $20 \mathrm{~m}$ & 0.52 & 36 & $T_{\mathrm{A}}^{*}$ & (1) \\
\hline IC 860 & $28-27$ & $0.54 \pm 0.07$ & $3.9 \pm 0.5$ & 175 & IRAM $30 \mathrm{~m}$ & 0.59 & 9 & $T_{\Delta}^{*}$ & (1) \\
\hline I17208 & $10-9$ & $0.33 \pm 0.03$ & $2.2 \pm 0.2$ & 330 & IRAM $30 \mathrm{~m}$ & 0.80 & 28 & $T_{\triangle}^{*}$ & (1) \\
\hline M82 & $12-11$ & $5.6 \pm 0.6$ & $30 \pm 3$ & 155 & IRAM $30 \mathrm{~m}$ & 0.80 & 25 & $T_{\mathrm{R}}^{*}$ & (3) \\
\hline Maffei 2 & $12-11$ & $1.42 \pm 0.05$ & $10.9 \pm 0.4$ & 200 & IRAM $30 \mathrm{~m}$ & 0.73 & 24 & $T_{\mathrm{A}}^{*}$ & (1) \\
\hline NGC 34 & $10-9^{b}$ & $<0.45$ & $<12$ & $\ldots$ & SEST $15 \mathrm{~m}$ & 0.75 & 55 & $T_{\mathrm{A}}^{*}$ & (1) \\
\hline NGC 253 & $9-8$ & $5.8 \pm 0.6$ & $27 \pm 3$ & $63^{c}$ & IRAM $30 \mathrm{~m}$ & $\ldots$ & 29 & $T_{\mathrm{mb}}$ & (4) \\
\hline NGC 253 & $10-9$ & $5.8 \pm 0.6$ & $27 \pm 3$ & $63^{c}$ & IRAM $30 \mathrm{~m}$ & $\ldots$ & 26 & $T_{\mathrm{mb}}$ & (4) \\
\hline NGC 253 & $12-11$ & $4.4 \pm 0.7$ & $19 \pm 3$ & $63^{c}$ & IRAM $30 \mathrm{~m}$ & $\ldots$ & 21 & $T_{\mathrm{mb}}$ & (4) \\
\hline NGC 253 & $15-14$ & $4.4 \pm 0.3$ & $24 \pm 2$ & 77,85 & IRAM $30 \mathrm{~m}$ & $\ldots$ & 19 & $T_{\mathrm{mb}}$ & (5) \\
\hline NGC 253 & $15-14$ & $3.6 \pm 0.6$ & $16 \pm 3$ & $63^{c}$ & IRAM $30 \mathrm{~m}$ & $\ldots$ & 17 & $T_{\mathrm{mb}}$ & (4) \\
\hline NGC 253 & $16-15$ & 3.8 & 19 & $77^{c}, 85^{c}$ & IRAM $30 \mathrm{~m}$ & $\ldots$ & 17 & $T_{\mathrm{mb}}$ & (5) \\
\hline NGC 253 & $17-16$ & $3.0 \pm 0.2$ & $15 \pm 1$ & $72^{c}$ & IRAM $30 \mathrm{~m}$ & $\ldots$ & 16 & $T_{\mathrm{mb}}$ & (5) \\
\hline NGC 253 & $17-16$ & $3.4 \pm 0.4$ & $15 \pm 2$ & $63^{c}$ & IRAM $30 \mathrm{~m}$ & $\ldots$ & 15 & $T_{\mathrm{mb}}$ & (4) \\
\hline NGC 253 & $18-17$ & $2.2 \pm 0.5$ & $11 \pm 2$ & 73 & IRAM $30 \mathrm{~m}$ & $\ldots$ & 15 & $T_{\mathrm{mb}}$ & (5) \\
\hline NGC 253 & $19-18$ & $4.6 \pm 0.6$ & $22 \pm 3$ & 74 & IRAM $30 \mathrm{~m}$ & $\ldots$ & 14 & $T_{\mathrm{mb}}$ & (5) \\
\hline NGC 253 & $26-25$ & $3.2 \pm 0.7$ & $21 \pm 5$ & $63^{c}$ & IRAM $30 \mathrm{~m}$ & $\ldots$ & 12 & $T_{\mathrm{mb}}$ & (4) \\
\hline NGC 613 & $10-9^{b}$ & $<0.26$ & $<7.0$ & $\ldots$ & SEST $15 \mathrm{~m}$ & 0.75 & 55 & $T_{\mathrm{A}}^{*}$ & (1) \\
\hline NGC 1056 & $16-15$ & $<0.17$ & $<1.3$ & $\ldots$ & IRAM $30 \mathrm{~m}$ & 0.67 & 17 & $T_{\mathrm{A}}^{*}$ & (1) \\
\hline NGC 1068 & $10-9^{b}$ & $0.39 \pm 0.05$ & $11 \pm 1$ & 100 & SEST $15 \mathrm{~m}$ & 0.75 & 55 & $T_{\mathrm{A}}^{*}$ & $(1)^{d}$ \\
\hline NGC 1365 & $10-9^{b}$ & $<0.61$ & $<417$ & $\ldots$ & SEST $15 \mathrm{~m}$ & 0.75 & 55 & $T_{\mathrm{A}}^{*}$ & $(1)^{d}$ \\
\hline NGC 1377 & $16-15$ & $<0.28$ & $<2.1$ & $\ldots$ & IRAM $30 \mathrm{~m}$ & 0.67 & 17 & $T_{\mathrm{A}}^{*}$ & (1) \\
\hline NGC 1377 & $25-24$ & $<0.26$ & $<1.9$ & $\ldots$ & IRAM $30 \mathrm{~m}$ & 0.63 & 10.5 & $T_{\mathrm{A}}^{*}$ & (1) \\
\hline NGC 1614 & $10-9^{b}$ & $<0.39$ & $<11$ & $\ldots$ & SEST $15 \mathrm{~m}$ & 0.75 & 55 & $T_{\mathrm{A}}^{*}$ & (1) \\
\hline NGC 1808 & $10-9^{b}$ & $0.2 \pm 0.1$ & $6 \pm 3$ & 250 & SEST $15 \mathrm{~m}$ & 0.75 & 57 & $T_{\mathrm{A}}^{*}$ & (2) \\
\hline NGC 2146 & $10-9$ & $<0.38$ & $<7.7$ & $\ldots$ & OSO $20 \mathrm{~m}$ & 0.59 & 42 & $T_{\mathrm{A}}^{*}$ & (1) \\
\hline NGC 2146 & $12-11$ & $<0.38$ & $<9.2$ & $\ldots$ & OSO $20 \mathrm{~m}$ & 0.52 & 36 & $T_{\mathrm{A}}^{*}$ & (1) \\
\hline NGC 2623 & $12-11$ & $<0.50$ & $<12$ & $\ldots$ & OSO $20 \mathrm{~m}$ & 0.52 & 36 & $T_{\mathrm{A}}^{*}$ & (1) \\
\hline NGC 3079 & $10-9$ & $0.60 \pm 0.05$ & $4.0 \pm 0.3$ & 500 & IRAM $30 \mathrm{~m}$ & 0.80 & 28 & $T_{\mathrm{A}}^{*}$ & (1) \\
\hline NGC 3079 & $16-15$ & $<0.54$ & $<4$ & $\ldots$ & IRAM $30 \mathrm{~m}$ & 0.80 & 17 & $T_{\mathrm{A}}^{*}$ & (1) \\
\hline NGC 3079 & $25-24$ & $<0.40$ & $<3$ & $\ldots$ & IRAM $30 \mathrm{~m}$ & 0.80 & 10.5 & $T_{\mathrm{A}}^{*}$ & (1) \\
\hline NGC 3256 & $10-9^{b}$ & $<0.12$ & $<3.3$ & $\ldots$ & SEST $15 \mathrm{~m}$ & 0.75 & 55 & $T_{\mathrm{A}}^{*}$ & (2) \\
\hline NGC 3690 & $12-11$ & $<0.31$ & $<7.5$ & $\ldots$ & OSO $20 \mathrm{~m}$ & 0.52 & 36 & $T_{\mathrm{A}}^{*}$ & (1) \\
\hline NGC $4418^{e}$ & $10-9$ & $0.8 \pm 0.08$ & $5 \pm 0.5$ & 122 & IRAM $30 \mathrm{~m}$ & 0.77 & 27 & $T_{\mathrm{A}}^{*}$ & (6) \\
\hline NGC $4418^{e}$ & $16-15^{f}$ & $1.7 \pm 0.08$ & $12 \pm 0.6$ & 130 & IRAM $30 \mathrm{~m}$ & 0.70 & 17 & $T_{\mathrm{A}}^{*}$ & (6) \\
\hline NGC $4418^{e}$ & $25-24$ & $1.6 \pm 0.2$ & $15 \pm 2$ & 140 & IRAM $30 \mathrm{~m}$ & 0.53 & 11 & $T_{\mathrm{A}}^{*}$ & (6) \\
\hline NGC 4945 & $9-8$ & $2.16 \pm 0.50$ & $47 \pm 11$ & 230 & SEST $15 \mathrm{~m}$ & 0.78 & 63 & $T_{\mathrm{mb}}$ & (7) \\
\hline NGC 4945 & $10-9$ & $1.99 \pm 0.21$ & $41 \pm 4$ & 290 & SEST $15 \mathrm{~m}$ & 0.75 & 55 & $T_{\mathrm{mb}}$ & (7) \\
\hline NGC 4945 & $11-10$ & $2.92 \pm 0.35$ & $65 \pm 8$ & 340 & SEST $15 \mathrm{~m}$ & 0.73 & 52 & $T_{\mathrm{mb}}$ & (7) \\
\hline NGC 4945 & $12-11$ & $4.18 \pm 0.38$ & $98 \pm 9$ & 340 & SEST $15 \mathrm{~m}$ & 0.71 & 49 & $T_{\mathrm{mb}}$ & (7) \\
\hline NGC 4945 & $15-14$ & $2.13 \pm 0.29$ & $52 \pm 7$ & 250 & SEST $15 \mathrm{~m}$ & 0.65 & 40 & $T_{\mathrm{mb}}$ & (7) \\
\hline NGC 4945 & $16-15^{g}$ & $5.02 \pm 0.19$ & $120 \pm 5$ & 330 & SEST $15 \mathrm{~m}$ & 0.63 & 37 & $T_{\mathrm{mb}}$ & (7) \\
\hline NGC 4945 & $17-16$ & $2.26 \pm 0.55$ & $48 \pm 12$ & 280 & SEST $15 \mathrm{~m}$ & 0.61 & 33 & $T_{\mathrm{mb}}$ & (7) \\
\hline NGC 4945 & $24-23$ & $<0.60$ & $<12$ & $\ldots$ & SEST $15 \mathrm{~m}$ & 0.48 & 23 & $T_{\mathrm{mb}}$ & (7) \\
\hline NGC 4945 & $25-24$ & $<0.60$ & $<12$ & $\ldots$ & SEST $15 \mathrm{~m}$ & 0.46 & 22 & $T_{\mathrm{mb}}$ & (7) \\
\hline NGC 5135 & $10-9^{b}$ & $<0.13$ & $<3.7$ & $\ldots$ & SEST $15 \mathrm{~m}$ & 0.75 & 55 & $T_{\mathrm{A}}^{*}$ & (1) \\
\hline NGC 6946 & $12-11$ & $<0.32$ & $<2.5$ & $\ldots$ & IRAM $30 \mathrm{~m}$ & 0.73 & 24 & $T_{\mathrm{A}}^{*}$ & (1) \\
\hline NGC 7130 & $10-9^{b}$ & $<0.10$ & $<2.7$ & $\ldots$ & SEST $15 \mathrm{~m}$ & 0.75 & 55 & $T_{\mathrm{A}}^{*}$ & (2) \\
\hline UGC 5101 & $10-9$ & $<0.12$ & $<0.76$ & $\ldots$ & IRAM $30 \mathrm{~m}$ & 0.80 & 28 & $T_{A}^{*}$ & (1) \\
\hline
\end{tabular}

References. (1) This work; (2) Aalto et al. (2002); (3) Henkel et al. (1988); (4) Mauersberger et al. (1990); (5) Martín et al. (2006); (6) Aalto et al. (2007); (7) Wang et al. (2004).

Notes. ${ }^{(a)}$ The temperature scale of the integrated intensities are given in the $T$ scale column. Upper limits are $2 \sigma$ calculated from the rms of the noise surrounding the line for our data. For our data, errors are given in $1 \sigma$ and calculated from the rms. Since many articles lack information about sizes of errors and methods used when calculating the errors, most errors are given as printed in the respective article. However, if the size of the error is clearly written, it has been recalculated to $1 \sigma .{ }^{(b)}$ Measured in HNC 1-0 spectrum. ${ }^{(c)}$ Fixed when fitting Gaussian. ${ }^{(d)}$ These values are calculated from HNC 1-0 spectra already published in Pérez-Beaupuits et al. (2007). ${ }^{(e)}$ See Costagliola \& Aalto (2010) for an extensive survey of $\mathrm{HC}_{3} \mathrm{~N}$ in this galaxy, with data not included in this table. ${ }^{(f)}$ Contaminated by para- $\mathrm{H}_{2} \mathrm{CO}$, estimated to $20 \%$. The given value is only for the $\mathrm{HC}_{3} \mathrm{~N}$ component. ${ }^{(g)}$ Contaminated by para- $\mathrm{H}_{2} \mathrm{CO}$. 
Table 5. Data from HCN 1-0 observations.

\begin{tabular}{|c|c|c|c|c|c|c|c|c|}
\hline Galaxy & $\begin{array}{c}I(\mathrm{HCN}) 1-0^{a} \\
{\left[\mathrm{~K} \mathrm{~km} \mathrm{~s}^{-1}\right]}\end{array}$ & $\begin{array}{c}S_{v} \Delta v \\
{\left[\mathrm{Jy} \mathrm{km} \mathrm{s}^{-1}\right]}\end{array}$ & $\begin{array}{c}\Delta v \\
{\left[\mathrm{~km} \mathrm{~s}^{-1}\right]}\end{array}$ & Telescope & $\eta_{\mathrm{mb}}$ & $\begin{array}{l}\theta_{\mathrm{mb}} \\
{\left[{ }^{\prime \prime}\right]}\end{array}$ & $T$ scale $^{a}$ & References \\
\hline Arp 220 & $9.7 \pm 0.4$ & $57 \pm 2$ & 530 & IRAM $30 \mathrm{~m}$ & 0.82 & 29.5 & $T_{\mathrm{mb}}$ & (1) \\
\hline Circinus & $5.2 \pm 0.8$ & $110 \pm 20$ & 300 & SEST $15 \mathrm{~m}$ & 0.75 & 57 & $T_{\mathrm{mb}}$ & (2) \\
\hline IC 342 & $15.5 \pm 0.9$ & $36 \pm 2$ & $\ldots$ & NRO $45 \mathrm{~m}$ & 0.54 & 19 & $T_{\mathrm{mb}}$ & (3) \\
\hline IC 694 & $1.29 \pm 0.09$ & $8.4 \pm 0.6$ & $\ldots$ & IRAM $30 \mathrm{~m}$ & 0.82 & 28 & $T_{\mathrm{A}}^{*}$ & (4) \\
\hline IC 860 & $\ldots$ & $\ldots$ & $\ldots$ & & $\ldots$ & $\ldots$ & & $\mathrm{N} / \mathrm{A}^{b}$ \\
\hline I17208 & $2.19 \pm 0.16$ & $14 \pm 1$ & $\ldots$ & IRAM $30 \mathrm{~m}$ & 0.82 & 28 & $T_{\Delta}^{*}$ & (4) \\
\hline I17208 & $0.91 \pm 0.19$ & $34 \pm 7$ & 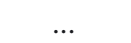 & NRAO $12 \mathrm{~m}$ & 0.89 & 72 & $T_{\mathrm{R}}^{\mathrm{A}}$ & (5) \\
\hline M82 & $29 \pm 0.2$ & $170 \pm 1$ & 130 & IRAM $30 \mathrm{~m}$ & 0.82 & 29.5 & $T_{\mathrm{mb}}^{\mathrm{K}}$ & (1) \\
\hline Maffei 2 & $13.8 \pm 0.9$ & $32 \pm 2$ & $\ldots$ & NRO $45 \mathrm{~m}$ & 0.54 & 19 & $T_{\mathrm{mb}}$ & (3) \\
\hline NGC 34 & $1.6 \pm 0.2$ & $33 \pm 4$ & $600-700$ & SEST $15 \mathrm{~m}$ & 0.75 & 57 & $T_{\mathrm{mb}}$ & (6) \\
\hline NGC 253 & 40.8 & 175 & 150 & NRO $45 \mathrm{~m}$ & 0.45 & 23 & $T_{A}^{*}$ & (7) \\
\hline NGC 613 & $0.53 \pm 0.08$ & $15 \pm 2$ & 130 & SEST $15 \mathrm{~m}$ & 0.75 & 57 & $T_{\Delta}^{*}$ & (8) \\
\hline NGC 1056 & $\ldots$ & $\ldots$ & $\ldots$ & & & $\ldots$ & & $\mathrm{N} / \mathrm{A}^{b}$ \\
\hline NGC 1068 & $24.5 \pm 0.9$ & $145 \pm 5$ & 220 & IRAM $30 \mathrm{~m}$ & 0.82 & 29.5 & $T_{\mathrm{mb}}$ & (1) \\
\hline NGC 1365 & $6.0 \pm 0.1$ & $125 \pm 2$ & $300-400$ & SEST $15 \mathrm{~m}$ & 0.75 & 57 & $T_{\mathrm{mb}}$ & (6) \\
\hline NGC 1377 & $0.47 \pm 0.1$ & $3.0 \pm 0.6$ & 140 & IRAM $30 \mathrm{~m}$ & 0.80 & 28 & $T_{\mathrm{A}}^{*}$ & (8) \\
\hline NGC 1614 & $1.5 \pm 0.22$ & $40 \pm 6$ & 300 & FCRAO $14 \mathrm{~m}$ & 0.60 & 50 & $T_{\mathrm{A}}^{*}$ & (5) \\
\hline NGC 1808 & 4 & 110 & & SEST $15 \mathrm{~m}$ & 0.74 & 56 & $T_{\mathrm{A}}^{\mathrm{A}}$ & (9) \\
\hline NGC 2146 & $5 \pm 0.1$ & $30 \pm 1$ & 290 & IRAM $30 \mathrm{~m}$ & 0.82 & 29.5 & $T_{\mathrm{mb}}^{\mathrm{A}}$ & (1) \\
\hline NGC 2623 & $\ldots$ & $\ldots$ & $\ldots$ & & & $\ldots$ & & $\mathrm{N} / \mathrm{A}^{b}$ \\
\hline NGC 3079 & $5.7 \pm 0.8$ & $29 \pm 4$ & 420 & IRAM $30 \mathrm{~m}$ & 0.80 & 28 & $T_{\mathrm{mb}}$ & (10) \\
\hline NGC 3079 & $2.6 \pm 0.42$ & $97 \pm 16$ & 365 & NRAO $12 \mathrm{~m}$ & 0.89 & 72 & $T_{\mathrm{R}}^{*}$ & (5) \\
\hline NGC 3256 & $2.3 \pm 0.2$ & $48 \pm 4$ & 165 & SEST $15 \mathrm{~m}$ & 0.77 & 57 & $T_{\mathrm{mb}}$ & (11) \\
\hline NGC 3690 & $2.04 \pm 0.11$ & $13.2 \pm 0.7$ & 300 & IRAM $30 \mathrm{~m}$ & 0.82 & 28 & $T_{\wedge}^{*}$ & (4) \\
\hline NGC 4418 & $1.96 \pm 0.04$ & $12.4 \pm 0.3$ & 170 & IRAM $30 \mathrm{~m}$ & 0.80 & 28 & $T_{\mathrm{A}}^{\mathrm{A}}$ & (8) \\
\hline NGC 4945 & $22.4 \pm 0.4$ & $436 \pm 8$ & 305 & SEST $15 \mathrm{~m}$ & 0.75 & 55 & $T_{\mathrm{mb}}^{\mathrm{A}}$ & (12) \\
\hline NGC 5135 & $0.65 \pm 0.07$ & $14 \pm 1.5$ & $50-60$ & SEST $15 \mathrm{~m}$ & 0.75 & 57 & $T_{\mathrm{mb}}$ & (6) \\
\hline NGC 6946 & $8.7 \pm 0.9$ & $20 \pm 2$ & $\ldots$ & NRO $45 \mathrm{~m}$ & 0.54 & 19 & $T_{\mathrm{mb}}$ & (3) \\
\hline NGC 7130 & $0.7 \pm 0.1$ & $15 \pm 2$ & 100 & SEST $15 \mathrm{~m}$ & 0.75 & 57 & $T_{\mathrm{mb}}$ & (6) \\
\hline UGC 5101 & $1.40 \pm 0.14$ & $9.1 \pm 0.9$ & 500 & IRAM $30 \mathrm{~m}$ & 0.82 & 28 & $T_{\wedge}^{*}$ & (4) \\
\hline
\end{tabular}

References. (1) Krips et al. (2008); (2) Curran et al. (2001a); (3) Sorai et al. (2002); (4) Graciá-Carpio et al. (2008); (5) Gao \& Solomon (2004); (6) Curran et al. (2000); (7) Nguyen-Q-Rieu et al. (1989); (8) This work; (9) Aalto et al. (1994); (10) Pérez-Beaupuits et al. (2007); (11) Casoli et al. (1992); (12) Wang et al. (2004).

Notes. ${ }^{(a)}$ The temperature scale of the integrated intensities are given in the $T$ scale column. Upper limits are $2 \sigma$ calculated from the rms of the noise surrounding the line for our data. For our data, errors are given in $1 \sigma$ and calculated from the rms. Since many articles lack information about sizes of errors and methods used when calculating the errors, most errors are given as printed in the respective article. However, if the size of the error is clearly written, it has been recalculated to $1 \sigma .{ }^{(b)}$ No single-dish HCN data were found in the literature for these objects.

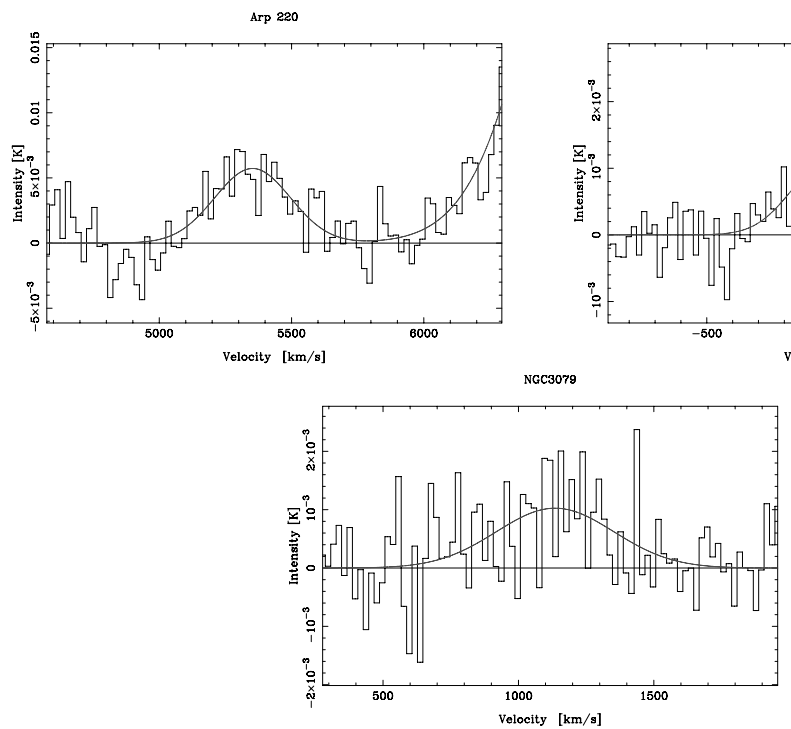

${ }^{\mathrm{I1}} 7208$

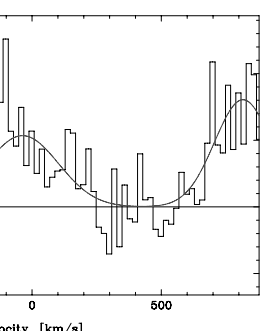

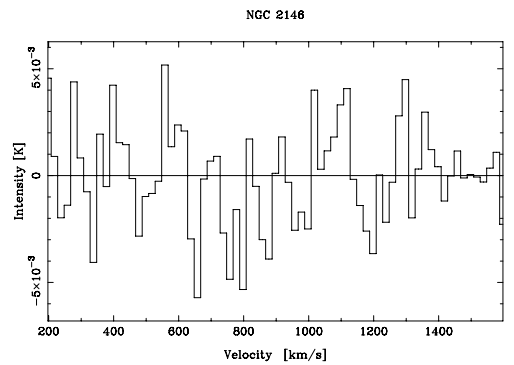

UGc5101

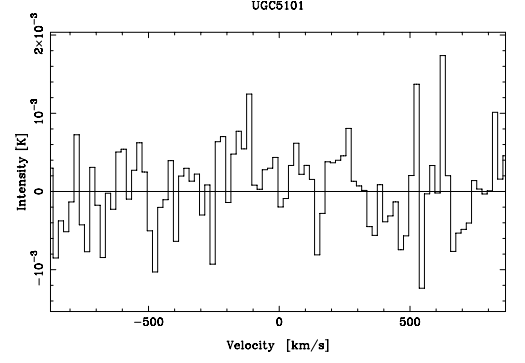

Fig. 1. $\mathrm{HC}_{3} \mathrm{~N}$ 10-9 spectra for Arp 220, IRAS 17208-0014 (detections), NGC 2146 (non-detection), NGC 3079 (detection), and UGC 5101 (nondetection). For Arp 220 and IRAS 17208-0014, part of the HNC 1-0 line is also visible. Some of the reported $\mathrm{HC}_{3} \mathrm{~N}$ 10-9-detections are found in the HNC 1-0 spectra, see Fig. 7. 
Table 6. Data from HNC 1-0 observations.

\begin{tabular}{|c|c|c|c|c|c|c|c|c|}
\hline Galaxy & $\begin{array}{c}I(\mathrm{HNC}) 1-0^{a} \\
{\left[\mathrm{~K} \mathrm{~km} \mathrm{~s}^{-1}\right]}\end{array}$ & $\begin{array}{c}S_{v} \Delta v \\
{\left[\mathrm{Jy} \mathrm{km} \mathrm{s}^{-1}\right]}\end{array}$ & $\begin{array}{c}\Delta v \\
{\left[\mathrm{~km} \mathrm{~s}^{-1}\right]}\end{array}$ & Telescope & $\eta_{\mathrm{mb}}$ & $\begin{array}{l}\theta_{\mathrm{mb}} \\
{\left[{ }^{\prime \prime}\right]}\end{array}$ & $T$ scale $^{a}$ & References \\
\hline Arp 220 & $0.95 \pm 0.2$ & $26 \pm 6$ & $\ldots$ & SEST $15 \mathrm{~m}$ & 0.75 & 55 & $T_{\mathrm{A}}^{*}$ & (1) \\
\hline Circinus & $1.99 \pm 0.1$ & $55 \pm 3$ & 280 & SEST $15 \mathrm{~m}$ & 0.75 & 55 & $T_{\mathrm{A}}^{*}$ & (2) \\
\hline IC 342 & $9.2 \pm 0.7$ & $48 \pm 4$ & 47 & IRAM 30 m & 0.8 & 25 & $T_{\mathrm{R}}^{*}$ & (3) \\
\hline IC 694 & $0.75 \pm 0.2$ & $15 \pm 4$ & $300-400$ & OSO $20 \mathrm{~m}$ & 0.59 & 42 & $T_{A}^{*}$ & (1) \\
\hline IC 860 & $0.70 \pm 0.04$ & $4.6 \pm 0.3$ & 230 & IRAM 30 m & 0.80 & 28 & $T_{A}^{*}$ & (2) \\
\hline I17208 & $1.12 \pm 0.06$ & $7.4 \pm 0.4$ & 350 & IRAM 30 m & 0.80 & 28 & $T_{\mathrm{A}}^{*}$ & (2) \\
\hline M82 & $7.3 \pm 0.6$ & $31 \pm 3$ & 129 & IRAM 30 m & 0.64 & 25 & $T_{\mathrm{mb}}^{\mathrm{A}}$ & (4) \\
\hline Maffei 2 & $7.3 \pm 0.8$ & $31 \pm 3$ & 91,50 & IRAM 30 m & 0.64 & 25 & $T_{\mathrm{mb}}$ & (4) \\
\hline NGC 34 & $<0.44$ & $<12$ & $\ldots$ & SEST $15 \mathrm{~m}$ & 0.75 & 55 & $T_{\mathrm{A}}^{*}$ & (2) \\
\hline NGC 253 & $50.0 \pm 2.8$ & $210 \pm 12$ & 72,136 & IRAM 30 m & 0.64 & 25 & $T_{\mathrm{mb}}^{\mathrm{A}}$ & (4) \\
\hline NGC 613 & $<0.24$ & $<6.5$ & $\ldots$ & SEST $15 \mathrm{~m}$ & 0.75 & 55 & $T_{\star}^{*}$ & (2) \\
\hline NGC 1056 & $<0.17$ & $<1.1$ & $\ldots$ & IRAM 30 m & 0.80 & 28 & $T_{A}^{*}$ & (2) \\
\hline NGC 1068 & $3.2 \pm 0.5$ & $65 \pm 10$ & 260 & SEST $15 \mathrm{~m}$ & 0.75 & 55 & $T_{\mathrm{mb}}^{\mathrm{A}}$ & (5) \\
\hline NGC 1068 & $11.4 \pm 0.7$ & $48 \pm 3$ & 232 & IRAM 30 m & 0.64 & 25 & $T_{\mathrm{mb}}$ & (4) \\
\hline NGC 1365 & $4.7 \pm 0.6$ & $96 \pm 12$ & 150 & SEST $15 \mathrm{~m}$ & 0.75 & 55 & $T_{\mathrm{mb}}$ & (5) \\
\hline NGC 1377 & $<0.15$ & $<0.99$ & $\ldots$ & IRAM 30 m & 0.80 & 28 & $T_{\mathrm{A}}^{*}$ & (2) \\
\hline NGC 1614 & $<0.38$ & $<10$ & $\ldots$ & SEST $15 \mathrm{~m}$ & 0.75 & 55 & $T_{\mathrm{A}}^{*}$ & (2) \\
\hline NGC 1808 & $1.2 \pm 0.1$ & $33 \pm 3$ & 300 & SEST $15 \mathrm{~m}$ & 0.75 & 55 & $T_{\mathrm{A}}^{*}$ & (1) \\
\hline NGC 2146 & $1.6 \pm 0.3$ & $6.7 \pm 1.3$ & 237 & IRAM 30 m & 0.64 & 25 & $T_{\mathrm{mb}}^{\mathrm{A}}$ & (4) \\
\hline NGC 2623 & $0.6 \pm 0.15$ & $12 \pm 3$ & $500-600$ & OSO 20 m & 0.59 & 42 & $T_{\mathrm{A}}^{*}$ & (1) \\
\hline NGC 3079 & $2.9 \pm 0.5$ & $15 \pm 3$ & 380 & IRAM 30 m & 0.80 & 28 & $T_{\mathrm{mb}}$ & (5) \\
\hline NGC 3079 & $6.9 \pm 1.0$ & $29 \pm 4$ & 545 & IRAM 30 m & 0.64 & 25 & $T_{\mathrm{mb}}$ & (4) \\
\hline NGC 3256 & $0.6 \pm 0.05$ & $16 \pm 1$ & 250 & SEST $15 \mathrm{~m}$ & 0.75 & 55 & $T_{\mathrm{A}}^{*}$ & (1) \\
\hline NGC 3690 & & $\cdots$ & & & & $\cdots$ & & $\mathrm{N} / \mathrm{A}^{b}$ \\
\hline NGC 4418 & $1.24 \pm 0.12$ & $7.9 \pm 0.8$ & 156 & IRAM 30 m & 0.77 & 27 & $T_{\mathrm{A}}^{*}$ & (6) \\
\hline NGC 4945 & $8.6 \pm 0.2$ & $230 \pm 5$ & 290 & SEST $15 \mathrm{~m}$ & 0.75 & 55 & $T_{\mathrm{A}}^{*}$ & (2) \\
\hline NGC 5135 & $<0.13$ & $<3.5$ & $\ldots$ & SEST $15 \mathrm{~m}$ & 0.75 & 55 & $T_{\mathrm{A}}^{*}$ & (2) \\
\hline NGC 6946 & $4.0 \pm 0.3$ & $17 \pm 1$ & 138 & IRAM 30 m & 0.64 & 25 & $T_{\mathrm{mb}}$ & (4) \\
\hline NGC 7130 & $0.4 \pm 0.05$ & $11 \pm 1$ & & SEST $15 \mathrm{~m}$ & 0.75 & 55 & $T_{\mathrm{A}}^{*}$ & (1) \\
\hline UGC 5101 & $1.24 \pm 0.1$ & $8.2 \pm 0.7$ & 500 & IRAM 30 m & 0.80 & 28 & $T_{\mathrm{A}}^{*}$ & (2) \\
\hline
\end{tabular}

References. (1) Aalto et al. (2002); (2) This work; (3) Henkel et al. (1988); (4) Hüttemeister et al. (1995); (5) Pérez-Beaupuits et al. (2007); (6) Aalto et al. (2007).

Notes. ${ }^{(a)}$ The temperature scale of the integrated intensities are given in the $T$ scale column. Upper limits are $2 \sigma$ calculated from the rms of the noise surrounding the line for our data. For our data, errors are given in $1 \sigma$ and calculated from the rms. Since many articles lack information about sizes of errors and methods used when calculating the errors, most errors are given as printed in the respective article. However, if the size of the error is clearly written, it has been recalculated to $1 \sigma .{ }^{(b)}$ No HNC data were found in the literature for this object.

\subsection{New detections}

This is not only the first text to put together a survey of all extragalactic $\mathrm{HC}_{3} \mathrm{~N}$ emission line data, but it also reports the first $\mathrm{HC}_{3} \mathrm{~N}$ detections in six galaxies: Circinus, IC 860, IRAS 17208-0014, Maffei 2, NGC 1068, and NGC 3079. The number of extragalactic sources where $\mathrm{HC}_{3} \mathrm{~N}$ has been detected is thus almost doubled. Three of the HNC detections are also made in sources without earlier HNC detections: Circinus, IC 860, and IRAS 17208-0014. Finally, the first detection of $\mathrm{HCN}$ in NGC 613 is also reported.

\subsection{Line ratios}

The line ratios have been calculated using the method described in Appendix A, and are shown in Table 7. As already mentioned, the $\mathrm{HC}_{3} \mathrm{~N} / \mathrm{HCN}$ and $\mathrm{HC}_{3} \mathrm{~N} / \mathrm{HNC}$ line ratios of some of the most nearby galaxies will be somewhat overestimated due to their source size being larger than the beam size of the telescope. See Appendix A.1 for a discussion on this subject. The galaxies in our survey that do not fulfil the criterion $\theta_{\mathrm{s}} \lesssim \theta_{\mathrm{mb}}$ are IC 342, M82, Maffei 2, and NGC 253, and we therefore expect the real $\mathrm{HC}_{3} \mathrm{~N} / \mathrm{HCN}$ and $\mathrm{HC}_{3} \mathrm{~N} / \mathrm{HNC}$ ratios to be somewhat lower for these galaxies.
In Table 7, preference has been given to $\mathrm{HC}_{3} \mathrm{~N} 10-9$ lines before other $\mathrm{HC}_{3} \mathrm{~N}$ lines. Only if no 10-9 line is available, another $\mathrm{HC}_{3} \mathrm{~N}$ line (specified in the footnotes) has been used for the ratios.

A few of the galaxies appear twice in Table 7. For these, several observations have been found for the same transition. As can be seen, the values of these observations do not always agree. If the $\mathrm{HC}_{3} \mathrm{~N}$ and $\mathrm{HNC}$ data are found in the same spectrum in one of the observations, preference has been given to this observation, as it will increase the accuracy on the $\mathrm{HC}_{3} \mathrm{~N} / \mathrm{HNC}$ ratio. In all other cases, the spectrum of each observation has been investigated (when available), and the values from the spectra with the lowest noise levels have been given priority and are put first in Table 7.

\subsection{1. $\mathrm{HC}_{3} \mathrm{~N}$-luminous galaxies}

A definition of an $\mathrm{HC}_{3} \mathrm{~N}$-luminous galaxy is now desirable. As $\mathrm{HCN}$ is the most common dense gas tracer, and also should be a more stable component of the dense gas than HNC, the $\mathrm{HC}_{3} \mathrm{~N} / \mathrm{HCN}$ ratios were decided to be used for this definition. It seems like most galaxies have $\mathrm{HC}_{3} \mathrm{~N} / \mathrm{HCN}$ ratios below 0.15 , with the exception for a few interesting galaxies. Thus, we consider in the rest of the paper that galaxies with $\frac{I\left(\mathrm{HC}_{3} \mathrm{~N}\right)}{I(\mathrm{HCN})}>0.15$ 

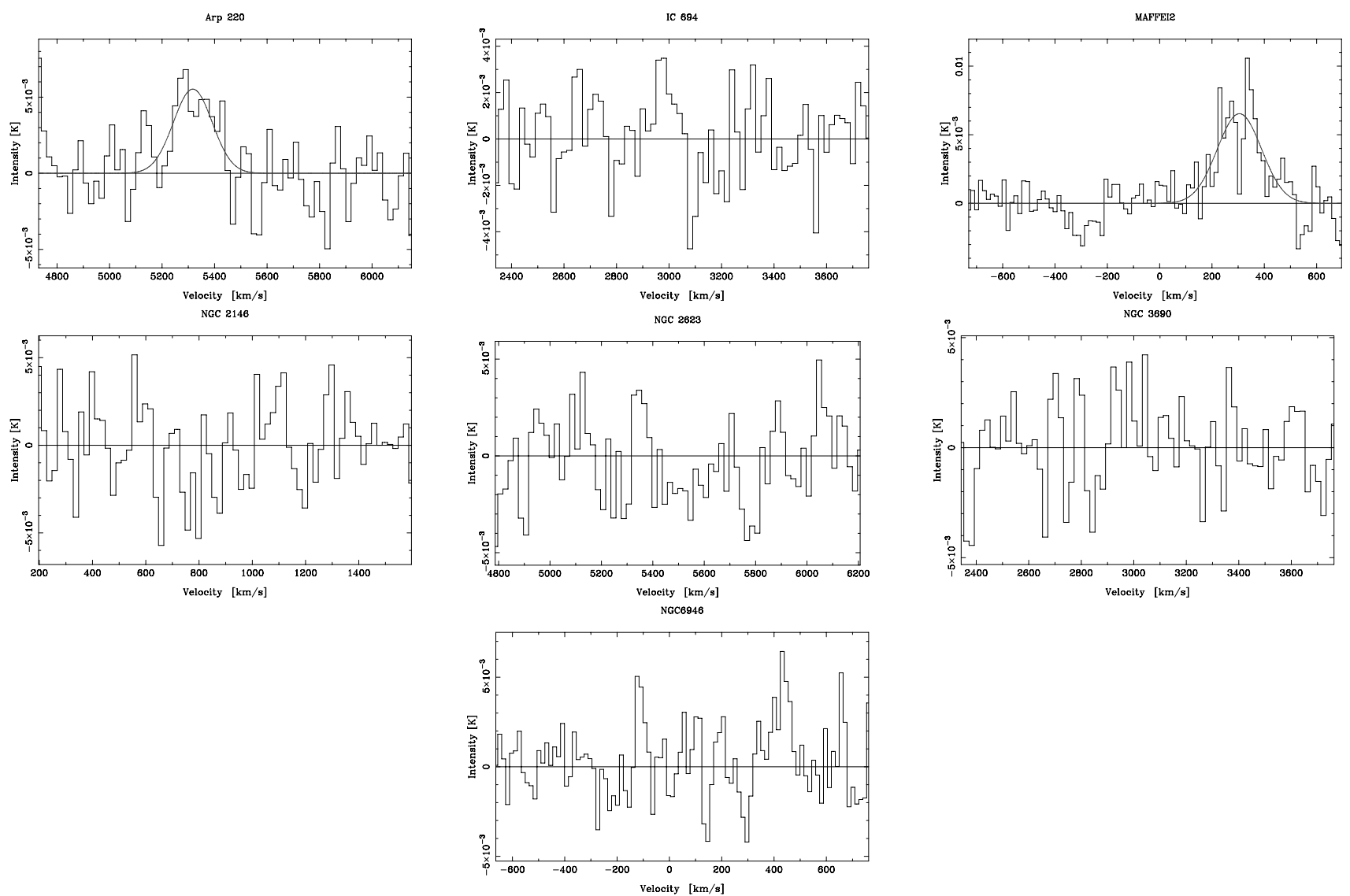

Fig. 2. $\mathrm{HC}_{3} \mathrm{~N}$ 12-11 spectra for Arp 220 (detection), IC 694 (non-detection), Maffei 2 (detection), NGC 2146, NGC 2623, NGC 3690, and NGC 6946 (non-detections).

NGC1056

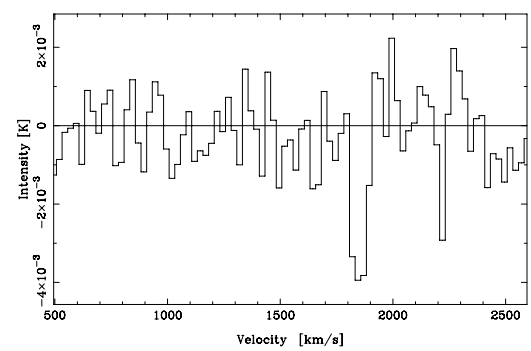

NGC1377

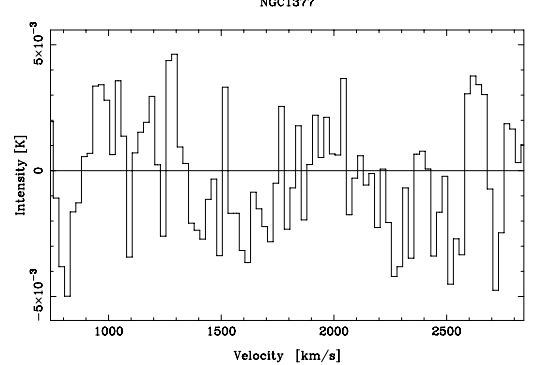

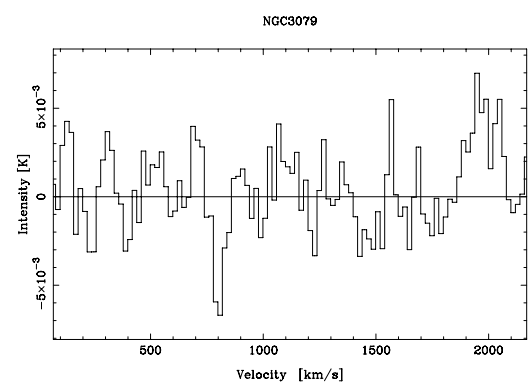

Fig. 3. $\mathrm{HC}_{3}$ N 16-15 spectra for NGC 1056, NGC 1377, and NGC 3079 (non-detections). The absorption-like features in the NGC 1056 spectrum are most likely due to a problem with the backend of the receiver.

are $\mathrm{HC}_{3} \mathrm{~N}$-luminous galaxies. If a corresponding limit should be set on the $\mathrm{HC}_{3} \mathrm{~N} / \mathrm{HNC}$ ratio, it would be around 0.25 to include the same galaxies.

The galaxies thus seen as $\mathrm{HC}_{3} \mathrm{~N}$-luminous or $\mathrm{HC}_{3} \mathrm{~N}$-rich are NGC 4418, IC 342, Circinus, M82, Maffei 2, Arp 220, and IRAS 17208-0014. We also choose to include IC 860, considering that its moderate $\mathrm{HC}_{3} \mathrm{~N} / \mathrm{HNC}$ ratio is for the $\mathrm{HC}_{3} \mathrm{~N}$ 28-27 transition, as the higher transitions seem to be weaker than the $J=10-9$ line in most galaxies where more than one line has been observed (when beam effects are compensated for). A few of these galaxies are quite nearby, and as discussed above, the $\mathrm{HC}_{3} \mathrm{~N} / \mathrm{HCN}$ ratio of galaxies with source sizes larger than the telescope beam size will probably be overestimated. This is particularly the case for M82, IC 342, and Maffei 2.

Some galaxies can definitely be seen as $\mathrm{HC}_{3} \mathrm{~N}$-poor, since they have $\mathrm{HC}_{3} \mathrm{~N} / \mathrm{HCN}$ ratios (or upper limits for this ratio) less than or equal to 0.10 : NGC 253, NGC 1068, NGC 1808 ,
NGC 3256, NGC 4945, NGC 6946, and UGC 5101. Since NGC 253 also belongs to the nearby galaxies, this value should probably be even lower.

\section{Discussion}

It is not surprising to find that most galaxies in our sample from published articles show $\mathrm{HC}_{3} \mathrm{~N}$ detections - otherwise they would not be submitted for publication. However, if only counting the galaxies first investigated by us, there are only $6 \mathrm{HC}_{3} \mathrm{~N}$ detections in the 19 galaxies. This sample of galaxies was nevertheless made to find a high number of $\mathrm{HC}_{3} \mathrm{~N}$-luminous galaxies. This could mean one of three things:

- Our search-criteria for $\mathrm{HC}_{3} \mathrm{~N}$-luminous galaxies are not appropriate.

- The limit for $\mathrm{HC}_{3} \mathrm{~N}$-luminous galaxies is set too high to include all "interesting" objects. 

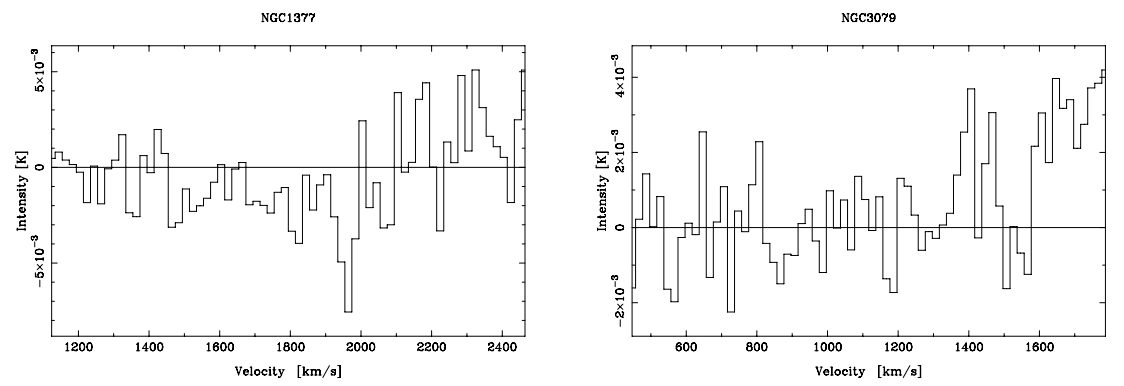

Fig. 4. $\mathrm{HC}_{3} \mathrm{~N} 25-24$ spectra for NGC 1377 and NGC 3079 (non-detections).

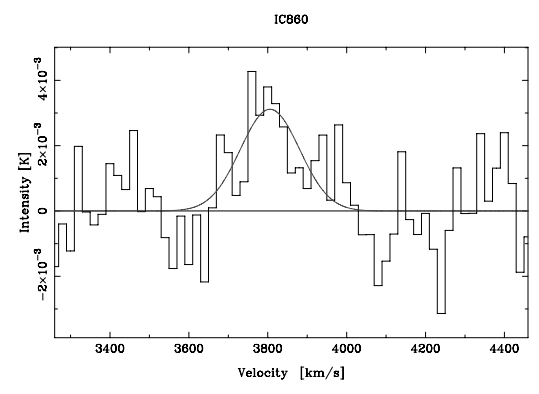

Fig. 5. $\mathrm{HC}_{3} \mathrm{~N} 28-27$ spectrum for IC 860 (detection).
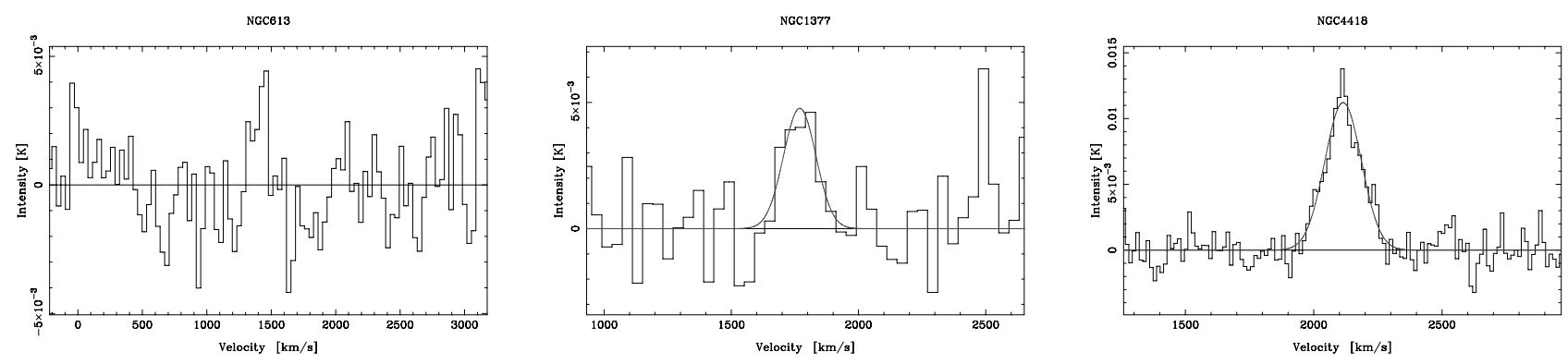

Fig. 6. HCN 1-0 spectra for NGC 613 (non-detection), NGC 1377, and NGC 4418 (detections).

- $\mathrm{HC}_{3} \mathrm{~N}$-luminous galaxies are very rare, even among active galaxies.

Several possible correlations between a high $\mathrm{HC}_{3} \mathrm{~N}$ intensity and other properties of the galaxies have been examined. This will be discussed below.

\subsection{In which types of galaxies do we find $\mathrm{HC}_{3} \mathrm{~N}$ ?}

If the $\mathrm{HC}_{3} \mathrm{~N}$-rich and -poor galaxies are compared with the galaxy classifications of Table 3, the most obvious trend is that most of the $\mathrm{HC}_{3} \mathrm{~N}$-poor galaxies are starbursts, with the exception for the LIRG UGC 5101. It is difficult to see any trend for the AGNs due to the low number of such objects in the sample.

When removing the nearby galaxies from the $\mathrm{HC}_{3} \mathrm{~N}$-rich category due to their overestimated $\mathrm{HC}_{3} \mathrm{~N} / \mathrm{HCN}$-ratios mentioned earlier, the common denominator of the remaining galaxies seems to be that their source of activity is unknown or disputed - they are labelled as "obscured" or ULIRGs. Thus, $\mathrm{HC}_{3} \mathrm{~N}$ might thrive in deeply obscured, shielded regions, where it cannot be destroyed by radiation. In starbursts, it might be destroyed by the strong UV field - or not even created, as $\mathrm{C}_{2} \mathrm{H}_{2}$ on the grains will photo-dissociate into $\mathrm{C}_{2} \mathrm{H}$ (see Sect. 2.1).

\subsection{Silicate absorption strength}

In Spoon et al. (2002), several absorption features from ice and silicates as well as emission from PAHs in active galaxies are discussed. In Spoon et al. (2007), an evolutionary plot for active galaxies is produced, showing two distinct regions in a plot over the equivalent width of the PAH $6.2 \mu \mathrm{m}$ emission line versus the strength of the silicate $9.7 \mu \mathrm{m}$ absorption band. Starburst galaxies tend to have a high PAH equivalent width, Seyfert galaxies have low PAH equivalent width and low silicate absorption strength, while ULIRGs have high silicate absorption strength and often also low PAH equivalent width.

By private communication with H. W. W. Spoon, the numerical values for silicate absorption strength in all the galaxies in his sample were obtained. Most galaxies in our sample are also included in his sample. When comparing these values to our $\mathrm{HC}_{3} \mathrm{~N} / \mathrm{HCN}$ ratios, a tentative pattern seems to appear.

In Fig. 8, the relation between the $\mathrm{HC}_{3} \mathrm{~N} / \mathrm{HCN}$ ratio and the silicate absorption strength is plotted. We note that two of the three nearby galaxies in the sample, M82 and IC 342, are showing too high $\mathrm{HC}_{3} \mathrm{~N} / \mathrm{HCN}$ ratios to fit into the pattern of the figure, which was expected (see Appendix A.1). A correlation seems possible when excluding the nearby M82, NGC 253, and IC 342 (correlation coefficient $r=-0.49$ ). One explanation to the correlation might be that $\mathrm{HC}_{3} \mathrm{~N}$ is formed in regions with silicates, where the silicates protect the $\mathrm{HC}_{3} \mathrm{~N}$ from radiation. 

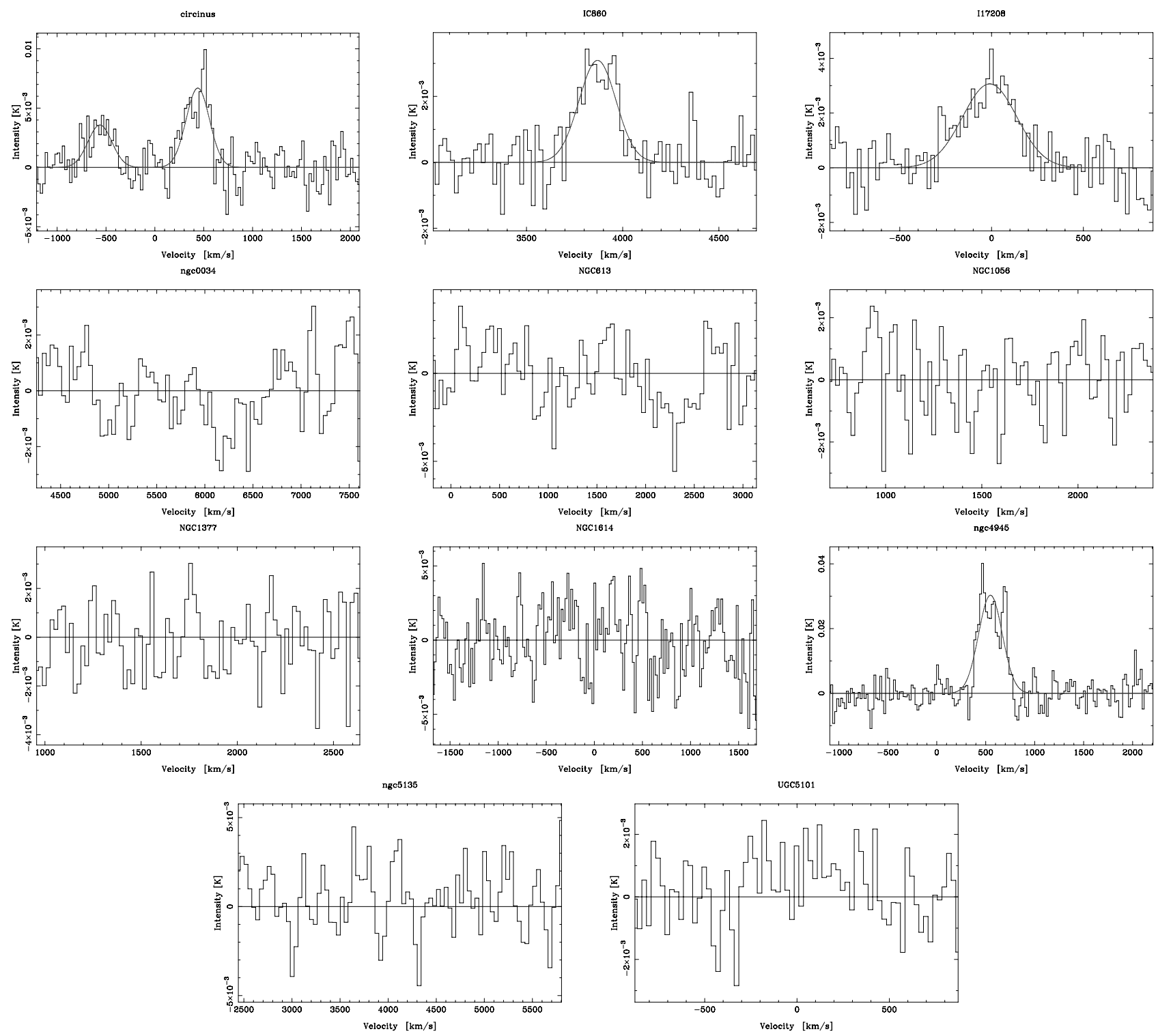

Fig. 7. HNC 1-0 spectra for Circinus, IC 860, IRAS 17208-0014 (detections), NGC 34, NGC 613, NGC 1056, NGC 1377, NGC 1614 (nondetections), NGC 4945 (detection), NGC 5135, and UGC 5101 (non-detections). For Circinus, the $\mathrm{HC}_{3} \mathrm{~N}$ 10-9 line is also visible. Bandwidths for the spectra of NGC 34, NGC 613, NGC 1614, and NGC 5135 are broad enough to include non-detections of the $\mathrm{HC}_{3} \mathrm{~N}$ 10-9 line, thus giving upper limits for these lines.

Thus, $\mathrm{HC}_{3} \mathrm{~N}$ survives better in regions heavily obscured by silicates.

\subsection{Megamasers}

Neglecting the most nearby galaxies, where the $\mathrm{HC}_{3} \mathrm{~N} / \mathrm{HCN}$ ratios probably are somewhat overestimated, all $\mathrm{HC}_{3} \mathrm{~N}$-luminous galaxies have $\mathrm{OH}$ mega- or kilomasers (Darling \& Giovanelli 2006). A few of the $\mathrm{HC}_{3} \mathrm{~N}$-poor galaxies also have $\mathrm{OH}$ mega- or kilomaser activity (NGC 253, NGC 1068, and UGC 5101). The concept of defining megamaser strength only from its luminosity is however somewhat misleading. Firstly, a galaxy with much molecular gas is more likely to harbour a strong megamaser than one with little molecular gas - the data in Darling (2007) show a strong correlation between $\mathrm{OH}$ megamaser strength and $\mathrm{CO}$ and HCN luminosity. Thus, it is more reasonable to normalise the $\mathrm{OH}$ megamaser luminosity with some kind of luminosity for the molecular gas in the galaxy. The CO 1-0 luminosity has been chosen for this, since $\mathrm{CO}$ is the primary tracer of molecular gas.

For IC 860, no CO 1-0 luminosity value has been found in the literature, but from an observation performed with the IRAM 30 m telescope by F. Costagliola the luminosity could be calculated from the intensity with the method described in Solomon et al. (1997). The used equation is:

$L_{\mathrm{CO}}=23.5 \Omega_{s * b} D_{L}^{2} I_{\mathrm{CO}}(1+z)^{-3}$

where $L_{\mathrm{CO}}$ is the line luminosity in $\mathrm{K} \mathrm{km} \mathrm{s}^{-1} \mathrm{pc}^{2}, \Omega_{s * b}$ is the solid angle of the source convolved with the telescope beam in $\operatorname{arcsec}^{2}, D_{L}$ is the luminosity distance in Mpc, $I_{\mathrm{CO}}$ is the main beam intensity of the line in $\mathrm{K} \mathrm{km} \mathrm{s}^{-1}$, and $z$ is the redshift of the source. The intensity $9.83 \mathrm{~K} \mathrm{~km} \mathrm{~s}^{-1}$, the beam width $22^{\prime \prime}$, and the distance $59.1 \mathrm{Mpc}$ gives a luminosity of $3.07 \times 10^{8} \mathrm{~K} \mathrm{~km} \mathrm{~s}^{-1} \mathrm{pc}^{2}$, assuming that the source size is much smaller than the beam size, which is valid for IC 860 . 
Table 7. Calculated line ratios.

\begin{tabular}{lccc}
\hline \hline Galaxy & $\frac{I\left(\mathrm{HC}_{3} \mathrm{~N}\right)}{I(\mathrm{HCN} 1-0)}$ & $\frac{I\left(\mathrm{HC}_{3} \mathrm{~N}\right)}{I(\mathrm{HNC} 1-0)}$ & $\frac{I(\mathrm{HNC} 1-0)}{I(\mathrm{HCN} 1-0)}$ \\
\hline Arp 220 & $0.19 \pm 0.07^{a}$ & $0.42 \pm 0.2^{a}$ & $0.45 \pm 0.1$ \\
Arp 220 & $0.78 \pm 0.06^{b}$ & $1.73 \pm 0.4^{b}$ & $0.45 \pm 0.1$ \\
Circinus & $0.24 \pm 0.04$ & $0.51 \pm 0.06$ & $0.48 \pm 0.08$ \\
IC 342 & $0.28 \pm 0.08$ & $0.28 \pm 0.08$ & $1.00 \pm 0.1$ \\
IC 694 & $<0.61^{c}$ & $<0.34^{c}$ & $1.79 \pm 0.5$ \\
IC 860 & $\ldots$ & $0.11 \pm 0.02^{d}$ & $\ldots$ \\
I17208 & $0.16 \pm 0.02^{e}$ & $0.30 \pm 0.03$ & $0.53 \pm 0.05^{e}$ \\
I17208 & $0.062 \pm 0.01^{f}$ & $0.30 \pm 0.03$ & $0.21 \pm 0.05^{f}$ \\
M82 & $0.21 \pm 0.02^{c}$ & $0.96 \pm 0.1^{c}$ & $0.22 \pm 0.02$ \\
Maffei 2 & $0.20 \pm 0.02^{c}$ & $0.25 \pm 0.03^{c}$ & $0.81 \pm 0.1$ \\
NGC 34 & $<0.35$ & $\ldots$ & $<0.34$ \\
NGC 253 & $0.094 \pm 0.01$ & $0.15 \pm 0.02$ & $0.63 \pm 0.04$ \\
NGC 613 & $<0.46$ & $\ldots$ & $<0.44$ \\
NGC 1056 & $\ldots$ & $\ldots$ & $\ldots$ \\
NGC 1068 & $0.072 \pm 0.06$ & $0.15 \pm 0.15^{g}$ & $0.45 \pm 0.19^{g}$ \\
NGC 1365 & $<0.13$ & $<0.17$ & $0.74 \pm 0.20$ \\
NGC 1377 & $<0.26^{h}$ & $\ldots$ & $<0.32$ \\
NGC 1614 & $<0.25$ & $\ldots$ & $<0.24$ \\
NGC 1808 & $0.05 \pm 0.025$ & $0.18 \pm 0.09$ & $0.29 \pm 0.02$ \\
NGC 2146 & $<0.20$ & $<0.85$ & $0.26 \pm 0.06$ \\
NGC 2623 & $\ldots$ & $<0.70^{c}$ & $\ldots$ \\
NGC 3079 & $0.13 \pm 0.02^{i}$ & $0.26 \pm 0.05^{i}$ & $0.51 \pm 0.11^{i}$ \\
NGC 3256 & $<0.065$ & $<0.20$ & $0.32 \pm 0.04$ \\
NGC 3690 & $<0.39^{c}$ & $\ldots$ & $\ldots$ \\
NGC 4418 & $0.40 \pm 0.04$ & $0.65 \pm 0.09$ & $0.61 \pm 0.06$ \\
NGC 4945 & $0.09 \pm 0.01$ & $0.17 \pm 0.02$ & $0.51 \pm 0.02$ \\
NGC 5135 & $<0.26$ & $\ldots$ & $<0.25$ \\
NGC 6946 & $<0.073^{c}$ & $<0.10^{c}$ & $0.71 \pm 0.1$ \\
NGC 7130 & $<0.18$ & $<0.25$ & $0.71 \pm 0.1$ \\
UGC 5101 & $<0.084$ & $<0.09$ & $0.91 \pm 0.1$ \\
\hline & & &
\end{tabular}

Notes. The $\mathrm{HC}_{3} \mathrm{~N}$ line used is the $10-9$ line, whenever it is available. If another line is used, this is stated below. The errors are $1 \sigma$ and have been calculated by error propagation from the errors given in Tables 4-6. ${ }^{(a)} \mathrm{HC}_{3} \mathrm{~N}$ value from Aalto et al. (2002) used. ${ }^{(b)} \mathrm{HC}_{3} \mathrm{~N}$ value from this work used. ${ }^{(c)} \mathrm{HC}_{3} \mathrm{~N}$ 12-11 line. ${ }^{(d)} \mathrm{HC}_{3} \mathrm{~N}$ 28-27 line. (e) $\mathrm{HCN}$ value from Graciá-Carpio et al. (2008) used. ${ }^{(f)} \mathrm{HCN}$ value from Gao \& Solomon (2004) used. ${ }^{(g)} \mathrm{HNC}$ value from Pérez-Beaupuits et al. (2007) used. ${ }^{(h)} \mathrm{HC}_{3} \mathrm{~N}$ 16-15 line. ${ }^{(i)} \mathrm{HCN}$ and $\mathrm{HNC}$ values from Pérez-Beaupuits et al. (2007) used.

In Table 8, the $\mathrm{OH}$ megamaser luminosity is compared with the CO luminosity. The data in this table is also shown in the form of a histogram in Fig. 9. The non-detections of $\mathrm{OH}$ megamasers and $\mathrm{OH}$ absorbers reported in Darling (2007) are also listed, most of them being either $\mathrm{HC}_{3} \mathrm{~N}$-poor or without any $\mathrm{HC}_{3} \mathrm{~N}$ detection (the exceptions are IC 342 and M82, whose $\mathrm{HC}_{3} \mathrm{~N} / \mathrm{HCN}$ ratios probably are overestimated, see Appendix A.1).

It can be seen that the $\mathrm{OH}$ megamaser luminosities normalised with the galactic $\mathrm{CO}$ luminosities are much higher in the $\mathrm{HC}_{3} \mathrm{~N}$-luminous galaxies than in the $\mathrm{HC}_{3} \mathrm{~N}$-poor galaxies with an $\mathrm{OH}$ megamaser, especially when ignoring the nearby galaxies $\mathrm{M} 82$ and IC 342. The average of the $\mathrm{OH} / \mathrm{CO}$ luminosity ratios is more than 10 times higher in the $\mathrm{HC}_{3} \mathrm{~N}$-luminous galaxies than in the $\mathrm{HC}_{3} \mathrm{~N}$-poor galaxies. The non-detections (where $\mathrm{HC}_{3} \mathrm{~N}$ poor galaxies are overrepresented) are not included in these averages. UGC 5101 is the only $\mathrm{HC}_{3} \mathrm{~N}$-poor galaxy with an $\mathrm{OH}$ megamaser that is strong compared to the amount of molecular gas in the galaxy. Also without the $\mathrm{CO}$ normalisation the trend can be seen clearly.

A possible explanation of the $\mathrm{HC}_{3} \mathrm{~N}$ correlating with $\mathrm{OH}$ megamasers is that the $\mathrm{HC}_{3} \mathrm{~N}$ is protected against destructive

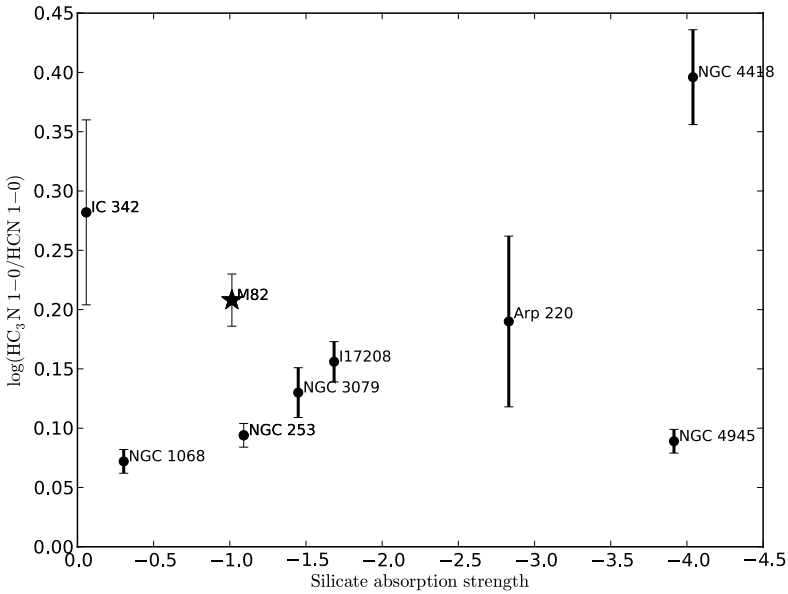

Fig. 8. Tentative correlation between $\mathrm{HC}_{3} \mathrm{~N} / \mathrm{HCN}$ ratio and silicate absorption strength. The $\mathrm{HC}_{3} \mathrm{~N} / \mathrm{HCN}$ values for IC 342 , NGC 253, and M82 are probably overestimated (indicated by thinner error bars, see Appendix A.1). The objects for which the $\mathrm{HC}_{3} \mathrm{~N}$ 12-11 line has been used instead of the $\mathrm{HC}_{3} \mathrm{~N}$ 10-9 line when calculating the $\mathrm{HC}_{3} \mathrm{~N} / \mathrm{HCN}$ ratio are indicated by a star. The silicate absorption strength is in a magnitude scale: A higher negative number means stronger silicate absorption.

Table 8. $\mathrm{OH}$ maser luminosities and $\mathrm{CO}$ luminosities for some of the sources in the sample.

\begin{tabular}{|c|c|c|c|}
\hline Galaxy & $\begin{array}{c}\log L_{\mathrm{OH}} \\
{\left[L_{\odot}\right]}\end{array}$ & $\begin{array}{c}L_{\mathrm{CO} 1-0} \\
{\left[10^{8} \mathrm{~K} \mathrm{~km} \mathrm{~s}^{-1} \mathrm{pc}^{2}\right]}\end{array}$ & $\begin{array}{c}\frac{\frac{L_{\mathrm{OH}}}{L_{\mathrm{CO} 1-0}}}{\frac{L_{\odot}}{10^{8} \mathrm{~K} \mathrm{~km} \mathrm{~s}^{-1} \mathrm{pc}^{2}}}\end{array}$ \\
\hline Arp 220 & 2.58 & 78.5 & 4.8 \\
\hline IC 342 & None & $\ldots$ & $\ldots$ \\
\hline IC 694/NGC 3690 & 1.38 & 29 & 0.83 \\
\hline IC 860 & $0.27^{a}$ & $3.07^{b}$ & 0.61 \\
\hline I17208 & 3.04 & 146.9 & 7.5 \\
\hline M82 & -1.7 & 5.7 & 0.0035 \\
\hline NGC 253 & -1.3 & 4.6 & 0.011 \\
\hline NGC 1068 & -0.3 & 20.7 & 0.024 \\
\hline NGC 1365 & None & $\ldots$ & $\ldots$ \\
\hline NGC 1614 & None & $\ldots$ & $\ldots$ \\
\hline NGC 2146 & None & $\ldots$ & $\ldots$ \\
\hline NGC 3079 & Abs. & $\ldots$ & $\ldots$ \\
\hline NGC 4418 & $0.04^{a}$ & $1.03^{c}$ & 1.1 \\
\hline NGC 4945 & Abs. & $\ldots$ & $\ldots$ \\
\hline NGC 5135 & None & $\ldots$ & $\ldots$ \\
\hline NGC 7130 & None & $\ldots$ & $\ldots$ \\
\hline UGC 5101 & 1.61 & 50.8 & 0.80 \\
\hline
\end{tabular}

Notes. Data are from Darling (2007) unless stated otherwise below. The note "None" in the $L_{\mathrm{OH}}$ column indicates that Darling (2007) has not detected any $\mathrm{OH}$ megamaser emission, and "Abs." that an $\mathrm{OH}$ absorber is detected. The names of the $\mathrm{HC}_{3} \mathrm{~N}$-luminous galaxies are written in boldface. The $\mathrm{HC}_{3} \mathrm{~N} / \mathrm{HCN}$ values for IC 342 and M82 are probably overestimated (see Appendix A.1). ${ }^{(a)}$ From Darling \& Giovanelli (2002). ${ }^{(b)}$ From observations by F. Costagliola. ${ }^{(c)}$ From Albrecht et al. (2007).

UV radiation by the warm dust which is needed to power the megamaser (Darling \& Giovanelli 2006). Another possibility is that the $\mathrm{HC}_{3} \mathrm{~N}$ is pumped by the IR field caused by the warm dust, which also pumps the $\mathrm{OH}$ megamaser. 


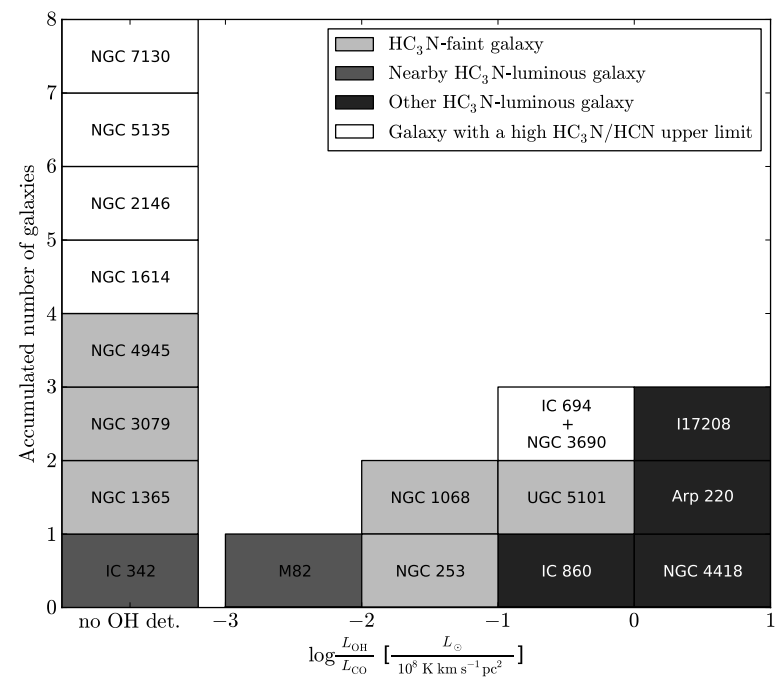

Fig. 9. Histogram over number of galaxies in normalised $\mathrm{OH}$ megamaser luminosity. Light-grey corresponds to galaxies considered $\mathrm{HC}_{3} \mathrm{~N}$-poor, medium-gray corresponds to nearby $\mathrm{HC}_{3} \mathrm{~N}$-luminous galaxies whose $\mathrm{HC}_{3} \mathrm{~N} / \mathrm{HCN}$ ratios probably are overestimated (see Appendix A.1), dark-grey corresponds to all other $\mathrm{HC}_{3} \mathrm{~N}$-luminous galaxies, and white corresponds to galaxies where the upper limit on the $\mathrm{HC}_{3} \mathrm{~N} / \mathrm{HCN}$ ratio is too high to tell whether it is $\mathrm{HC}_{3} \mathrm{~N}$-luminous or not. The galaxies for which $\mathrm{OH}$ maser emission is non-detected in Darling \& Giovanelli (2002) and/or Darling (2007) are stacked in the leftmost column of the histogram.

\subsection{IR flux density ratios}

In several of the $\mathrm{HC}_{3} \mathrm{~N}$ rich galaxies, rotational-vibrational $\mathrm{HC}_{3} \mathrm{~N}$ lines have been detected (Costagliola \& Aalto 2010; Martín et al. 2011; Costagliola et al. 2011). This suggests that IR pumping of the emission is present in these galaxies, which in turn indicates a warmer spectral energy distribution (SED) in these sources. We have thus compared the $\mathrm{HC}_{3} \mathrm{~N} / \mathrm{HCN}$ ratios with the IRAS $60 \mu \mathrm{m} / 100 \mu \mathrm{m}$ flux density ratios, but no linear correlation could be found. However, when plotting the data as a histogram (Fig. 10), we see a trend towards $\mathrm{HC}_{3} \mathrm{~N}$ luminous galaxies having higher IRAS $60 \mu \mathrm{m} / 100 \mu \mathrm{m}$ ratios, corresponding to warmer SEDs. NGC 4418, the object with the highest $\mathrm{HC}_{3} \mathrm{~N} / \mathrm{HCN}$ ratio in the sample is also the galaxy with the highest IRAS $60 \mu \mathrm{m} / 100 \mu \mathrm{m}$ ratio.

It should however be mentioned that the global $60 \mu \mathrm{m} / 100 \mu \mathrm{m}$ flux density ratios might not be completely relevant, since they may tell more about the temperature on the extended dust distribution (and/or foreground dust) than the dust temperature in the nucleus, where the pumping likely occurs.

\section{5. $\mathrm{C} \|$ flux}

Many of the objects with high $\mathrm{HC}_{3} \mathrm{~N} / \mathrm{HCN}$ ratios also have low $\mathrm{C}$ II/FIR flux ratios. This can be explained by that the $\mathrm{C}^{+}$ions are able to destroy $\mathrm{HC}_{3} \mathrm{~N}$ through reactions 5 and 6 in Sect. 2.2. We have searched in the literature for available $\mathrm{C}_{\text {II }}$ fluxes of the objects, which were found for more than half of the objects in our sample. With the limited amount of data, no linear correlation could be established. Instead, we display the data in the form of a histogram in Fig. 11, where we clearly see that a majority of the $\mathrm{HC}_{3} \mathrm{~N}$-rich galaxies are very poor in $\mathrm{C}$ II flux. We also see that the nearby galaxies for which the high $\mathrm{HC}_{3} \mathrm{~N} / \mathrm{HCN}$ ratio

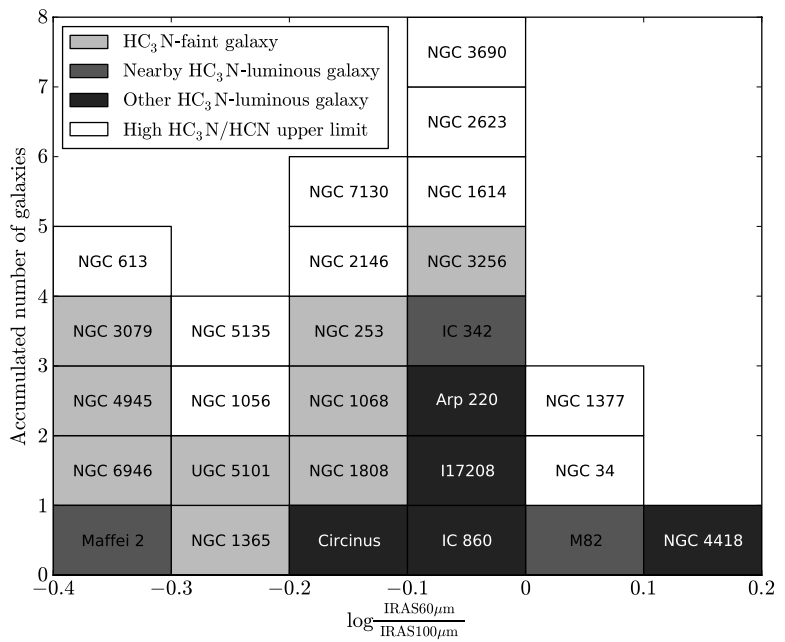

Fig. 10. Histogram over number of galaxies in IRAS $60 \mu \mathrm{m} / 100 \mu \mathrm{m}$ flux density ratio. Light-grey corresponds to galaxies considered $\mathrm{HC}_{3} \mathrm{~N}$-poor, medium-gray corresponds to nearby $\mathrm{HC}_{3} \mathrm{~N}$-luminous galaxies whose $\mathrm{HC}_{3} \mathrm{~N} / \mathrm{HCN}$ ratios probably are overestimated (see Appendix A.1), dark-grey corresponds to all other $\mathrm{HC}_{3} \mathrm{~N}$-luminous galaxies, and white corresponds to galaxies where the upper limit on the $\mathrm{HC}_{3} \mathrm{~N} / \mathrm{HCN}$ ratio is too high to tell whether it is $\mathrm{HC}_{3} \mathrm{~N}$-luminous or not. IRAS $60 \mu \mathrm{m} / 100 \mu \mathrm{m}$ flux density values are all from Sanders et al. (2003), except for Circinus and Maffei 2, which are from Beichman et al. (1988).

we expected to be an over-estimation due to beam effects (see Appendix A.1) all belong to the $\mathrm{C}_{\text {II }}$ rich part of the histogram. Also in this case, NGC 4418 is the most extreme galaxy, with the lowest upper limit on the $\mathrm{C}$ II/FIR flux ratio.

\section{6. $\mathrm{HNC/HCN}$}

The HNC/HCN 1-0 ratio is an indicator of the physical and chemical conditions in the dense molecular gas. Overluminous HNC is a sign of XDR chemistry (Aalto 2008), while a low $\mathrm{HNC} / \mathrm{HCN}$ ratio indicates shocks (Schilke et al. 1992). As is seen in Fig. 12, we find a correlation between the $\mathrm{HC}_{3} \mathrm{~N} / \mathrm{HCN}$ and $\mathrm{HNC} / \mathrm{HCN}$ 1-0 line ratios. The $\mathrm{HC}_{3} \mathrm{~N} / \mathrm{HCN}$ ratios of $\mathrm{M} 82$, Maffei 2, IC 342, and NGC 253 are probably overestimated (see Appendix A.1), and the correlation coefficient is $r=0.65$ when these four objects are excluded. This correlation will be further discussed in Costagliola et al. (2011).

Attempts were also made trying to find a correlation between the $\mathrm{HC}_{3} \mathrm{~N}$ ratios and ratios of higher $\mathrm{HNC}$ and $\mathrm{HCN}$ transitions, e.g. the $\mathrm{HNC} / \mathrm{HCN} 3-2$ ratio. However, too few HNC 3-2 and HCN 3-2 spectra for the galaxies in the sample are available in the literature - with only five data points no conclusions can be drawn. It was although noticed that the HCN 3-2 and HNC 3-2 intensities seem to be very uncertain, at least for Arp 220 and NGC 4418. Shortly, it seems like the different instruments used for the observations affect the measured value to a non-negligible extent. This is further discussed in Lindberg (2009).

We were not able to reproduce the weak correlation between the HNC/HCN 1-0 ratio and the FIR luminosity described in Aalto et al. (2002). 


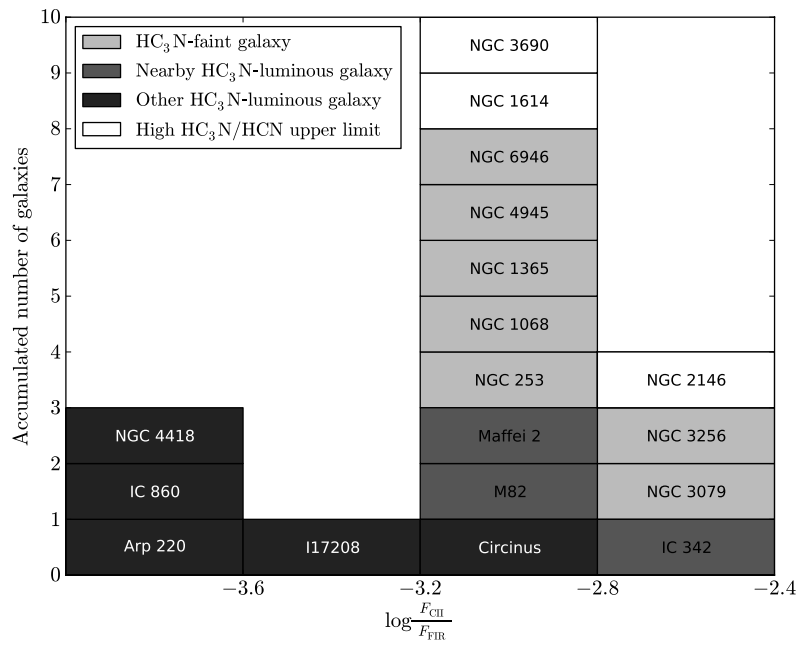

Fig. 11. Histogram over number of galaxies in $\mathrm{C}_{\text {II }}$ flux normalised by FIR flux. Light-grey corresponds to galaxies considered $\mathrm{HC}_{3} \mathrm{~N}$-poor, medium-gray corresponds to nearby $\mathrm{HC}_{3} \mathrm{~N}$-luminous galaxies whose $\mathrm{HC}_{3} \mathrm{~N} / \mathrm{HCN}$ ratios probably are overestimated (see Appendix A.1), dark-grey corresponds to all other $\mathrm{HC}_{3} \mathrm{~N}$-luminous galaxies, and white corresponds to galaxies where the upper limit on the $\mathrm{HC}_{3} \mathrm{~N} / \mathrm{HCN}$ ratio is too high to tell whether it is $\mathrm{HC}_{3} \mathrm{~N}$-luminous or not. FIR fluxes were calculated from IRAS $60 \mu \mathrm{m}$ and $100 \mu \mathrm{m}$ fluxes with the method described in Bizyaev (2001). The references for the used IRAS fluxes are given in the caption of Fig. 10. C II flux values are from Negishi et al. (2001), except for IC 342 and NGC 3079 (Stacey et al. 1991); IC 860 and NGC 4418 (Malhotra et al. 2001); Arp 220 (Luhman et al. 2003); and NGC 1614 (Brauher et al. 2009).

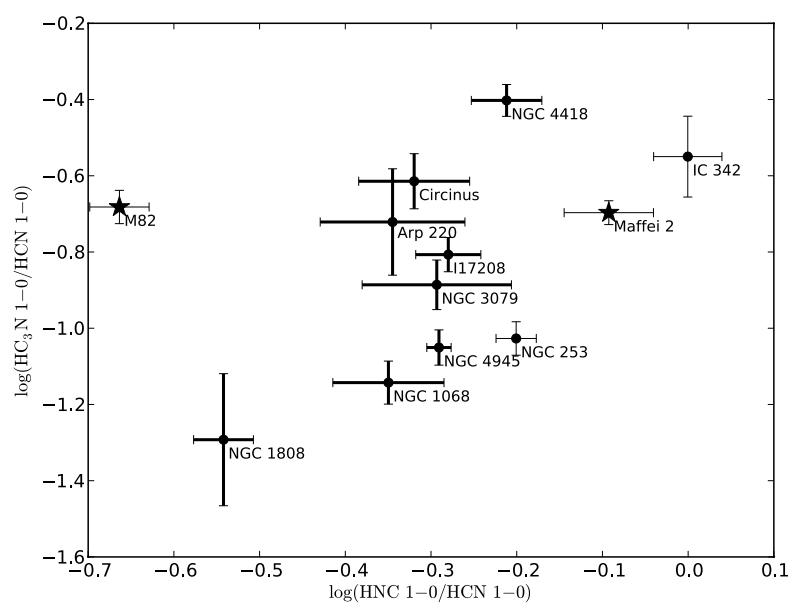

Fig. 12. log-log plot of the $\mathrm{HC}_{3} \mathrm{~N} / \mathrm{HCN}$ line ratio versus the $\mathrm{HNC} / \mathrm{HCN}$ line ratio. The $\mathrm{HC}_{3} \mathrm{~N} / \mathrm{HCN}$ values for IC 342, Maffei 2, M82, and NGC 253 are probably overestimated (indicated by thin error bars, see Appendix A.1). The objects for which the $\mathrm{HC}_{3} \mathrm{~N}$ 12-11 line has been used instead of the $\mathrm{HC}_{3} \mathrm{~N}$ 10-9 line when calculating the $\mathrm{HC}_{3} \mathrm{~N} / \mathrm{HCN}$ ratio are indicated by a star.

\subsection{Future observational tests}

We have found a strong trend between the $\mathrm{HC}_{3} \mathrm{~N} / \mathrm{HCN}$ ratio and $\mathrm{OH}$ megamaser activity (see Sect. 5.3). By studying different excitation levels of $\mathrm{HC}_{3} \mathrm{~N}$ in these and other $\mathrm{OH}$ megamaser galaxies, the cause of this correlation can be investigated. If $\mathrm{HC}_{3} \mathrm{~N}$ is pumped, higher transitions, including vibrational transitions, should be found. This has already been detected in NGC 4418 (Costagliola \& Aalto 2010).

To better establish the importance of $\mathrm{HC}_{3} \mathrm{~N}$ as an indicator of activity in certain galaxies, it is important to do further line surveys of $\mathrm{HC}_{3} \mathrm{~N}, \mathrm{HCN}$, and $\mathrm{HNC}$, as well as other molecules who trace the properties of the molecular gas, such as $\mathrm{HCO}^{+}$ and $\mathrm{C}_{2} \mathrm{H}$. Such a line survey could also test our weak correlation between the $\mathrm{HC}_{3} \mathrm{~N} / \mathrm{HCN}$ and $\mathrm{HNC} / \mathrm{HCN}$ line ratios, and perhaps finding other correlations which would enable a better understanding of the chemistry in obscured and active galaxies. A new line survey was made with the new EMIR receiver at the IRAM 30 m telescope in June 2009, and the results from this survey will be published in Costagliola et al. (2011). The sample in this survey has been chosen to get more $\mathrm{HC}_{3} \mathrm{~N}$ data on sources in Spoon's sample to test the possible correlations between the $\mathrm{HC}_{3} \mathrm{~N} / \mathrm{HCN}$ ratio and $\mathrm{PAH}$ equivalent width and/or silicate absorption strength. The bandwidth of the EMIR receiver allows for several spectral lines being observed in the same spectrum, and thus many different molecular species can be observed at the same time.

It might also be interesting to search for other long-carbonchain molecules in the $\mathrm{HC}_{3} \mathrm{~N}$-luminous galaxies, e.g. $\mathrm{HC}_{5} \mathrm{~N}$, $\mathrm{C}_{2} \mathrm{H}, \mathrm{C}_{4} \mathrm{H}, \mathrm{C}_{3} \mathrm{H}_{2}$, and $\mathrm{C}_{4} \mathrm{H}_{2}$, which have all been found in starforming regions in the Galaxy (Sakai et al. 2008, 2009).

Finally, we suggest mapping of $\mathrm{HC}_{3} \mathrm{~N}, \mathrm{C}_{2} \mathrm{H}, \mathrm{HCN}$, and $\mathrm{HNC}$ in a larger number of galaxies, especially in the $\mathrm{HC}_{3} \mathrm{~N}$-luminous galaxies, to compare the results with the maps of IC 342 in Meier \& Turner (2005). The $\mathrm{HC}_{3} \mathrm{~N}$ absorption lines detected in a $z \sim 0.89$ galaxy in front of PKS 1830-211 (Henkel et al. 2009) indicate that $\mathrm{HC}_{3} \mathrm{~N}$ is not only present in the core of a galaxy, but may also be present in the disc.

\section{Conclusions}

We have presented the first survey of $\mathrm{HC}_{3} \mathrm{~N}$ observations in extragalactic objects. The main conclusions from this survey are as follows:

1. Bright $\mathrm{HC}_{3} \mathrm{~N}$ emission is rather uncommon in galaxies. It was only detected in 6 of the 19 galaxies which had not been investigated before, even though that sample was selected to find many $\mathrm{HC}_{3} \mathrm{~N}$-luminous galaxies.

2. Most $\mathrm{HC}_{3} \mathrm{~N}$-luminous galaxies are obscured galaxies. Starburst galaxies seem to be poor in $\mathrm{HC}_{3} \mathrm{~N}$. There are too few AGN galaxies in the sample to tell if these normally are rich or poor in $\mathrm{HC}_{3} \mathrm{~N}$.

3. A weak correlation can be seen between the $\mathrm{HC}_{3} \mathrm{~N} / \mathrm{HCN}$ ratio and silicate $9.7 \mu \mathrm{m}$ absorption strength.

4. There is a strong correlation between $\mathrm{OH}$ megamaser activity and $\mathrm{HC}_{3} \mathrm{~N}$ luminosity. Most $\mathrm{HC}_{3} \mathrm{~N}$-luminous galaxies have an $\mathrm{OH}$ megamaser. This could be related to a high dust obscuration in the $\mathrm{HC}_{3} \mathrm{~N}$-luminous galaxies.

5. There is a connection between the $\mathrm{HC}_{3} \mathrm{~N} / \mathrm{HCN}$ ratio and the IRAS $60 \mu \mathrm{m} / 100 \mu \mathrm{m}$ flux density ratios, indicating a higher dust temperature in these galaxies, which could cause vibrational excitation of the $\mathrm{HC}_{3} \mathrm{~N}$ molecule.

6. There is a strong connection between a high $\mathrm{HC}_{3} \mathrm{~N} / \mathrm{HCN}$ ratio and a low $\mathrm{C}_{\mathrm{II}} / \mathrm{FIR}$ flux ratio in the studied objects. This could be explained by $\mathrm{C}^{+}$ions being required to destroy the $\mathrm{HC}_{3} \mathrm{~N}$ molecule.

7. There is a correlation between the $\mathrm{HC}_{3} \mathrm{~N} / \mathrm{HCN}$ and $\mathrm{HNC} / \mathrm{HCN}$ line ratios.

Acknowledgements. Many thanks to the IRAM, OSO, and SEST staff for their help during the observations. We are grateful to H. W. W. Spoon for sharing 
his PAH and silicate data with us. We would also like to thank the anonymous referee for several useful suggestions which improved the manuscript.

\section{References}

Aalto, S. 2008, Ap\&SS, 313, 273

Aalto, S., Booth, R. S., Black, J. H., Koribalski, B., \& Wielebinski, R. 1994, A\&A, 286, 365

Aalto, S., Booth, R. S., Black, J. H., \& Johansson, L. E. B. 1995, A\&A, 300, 369 Aalto, S., Radford, S. J. E., Scoville, N. Z., \& Sargent, A. I. 1997, ApJ, 475, L107

Aalto, S., Polatidis, A. G., Hüttemeister, S., \& Curran, S. J. 2002, A\&A, 381, 783

Aalto, S., Monje, R., \& Martín, S. 2007, A\&A, 475, 479

Albrecht, M., Krügel, E., \& Chini, R. 2007, A\&A, 462, 575

Baan, W. A., Henkel, C., Loenen, A. F., Baudry, A., \& Wiklind, T. 2008, A\&A, 477,747

Bajaja, E., Wielebinski, R., Reuter, H.-P., Harnett, J. I., \& Hummel, E. 1995, A\&AS, 114, 147

Beichman, C. A., Neugebauer, G., Habing, H. J., Clegg, P. E., \& Chester, T. J., 1988, Infrared astronomical satellite (IRAS) catalogs and atlases, Explanatory supplement, 1

Bizyaev, D. 2001, Ap\&SS, 276, 775

Bohme, D. K., \& Raksit, A. B. 1985, MNRAS, 213, 717

Brauher, J. R., Dale, D. A., \& Helou, G. 2009, VizieR Online Data Catalog, 217, 80280

Bryant, P. M., \& Scoville, N. Z. 1999, AJ, 117, 2632

Casoli, F., Dupraz, C., \& Combes, F. 1992, A\&A, 264, 49

Chapman, J. F., Millar, T. J., Wardle, M., Burton, M. G., \& Walsh, A. J. 2009, MNRAS, 394, 221

Cherchneff, I., Glassgold, A. E., \& Mamon, G. A. 1993, ApJ, 410, 188

Costagliola, F., \& Aalto, S. 2010, A\&A, 515, A71

Costagliola, F., Aalto, S., Rodriguez, M. I., et al. 2011, A\&A, in press

Curran, S. J., Aalto, S., \& Booth, R. S. 2000, A\&AS, 141, 193

Curran, S. J., Johansson, L. E. B., Bergman, P., Heikkilä, A., \& Aalto, S. 2001a, A\&A, 367, 457

Curran, S. J., Polatidis, A. G., Aalto, S., \& Booth, R. S. 2001b, A\&A, 373, 459

Dale, D. A., Sheth, K., Helou, G., Regan, M. W., \& Hüttemeister, S. 2005, AJ, 129,2197

\section{Darling, J. 2007, ApJ, 669, L9}

Darling, J., \& Giovanelli, R. 2002, AJ, 124, 100

Darling, J., \& Giovanelli, R. 2006, AJ, 132, 2596

de Vicente, P., Martín-Pintado, J., Neri, R., \& Colom, P. 2000, A\&A, 361, 1058

Fukuzawa, K., \& Osamura, Y. 1997, ApJ, 489, 113

Gao, Y., \& Solomon, P. M. 2004, ApJS, 152, 63

Graciá-Carpio, J., García-Burillo, S., Planesas, P., \& Colina, L. 2006, ApJ, 640, L135

Graciá-Carpio, J., García-Burillo, S., Planesas, P., Fuente, A., \& Usero, A. 2008, A\&A, 479, 703

Heikkilä, A., Johansson, L. E. B., \& Olofsson, H. 1999, A\&A, 344, 817
Henkel, C., Schilke, P., \& Mauersberger, R. 1988, A\&A, 201, L23

Henkel, C., Menten, K. M., Murphy, M. T., et al. 2009, A\&A, 500, 725

Hüttemeister, S., Henkel, C., Mauersberger, R., et al. 1995, A\&A, 295, 571

Imanishi, M., Nakanishi, K., Kuno, N., \& Kohno, K. 2004, AJ, 128, 2037

Irvine, W. M., Goldsmith, P. F., \& Hjalmarson, A. 1987, in Interstellar Processes, ed. D. J. Hollenbach \& H. A. Thronson, Jr., Astrophys. Space Sci. Libr., 134, 561

Knudsen, K. K., Walter, F., Weiss, A., et al. 2007, ApJ, 666, 156

Koda, J., Sofue, Y., Kohno, K., et al. 2002, ApJ, 573, 105

Kohno, K., Matsushita, S., Vila-Vilaró, B., et al. 2001, in The Central Kiloparsec of Starbursts and AGN: The La Palma Connection, ed. J. H. Knapen, J. E. Beckman, I. Shlosman, \& T. J. Mahoney, ASP Conf. Ser., 249, 672

Krips, M., Neri, R., García-Burillo, S., et al. 2008, ApJ, 677, 262

Lepp, S., \& Dalgarno, A. 1996, A\&A, 306, L21

Lindberg, J. 2009, Master's thesis, Chalmers Univ. Technol., Göteborg

Luhman, M. L., Satyapal, S., Fischer, J., et al. 2003, ApJ, 594, 758

Malhotra, S., Kaufman, M. J., Hollenbach, D., et al. 2001, ApJ, 561, 766

Maloney, P. R., Hollenbach, D. J., \& Tielens, A. G. G. M. 1996, ApJ, 466, 561

Martín, S., Krips, M., Martín-Pintado, J., et al. 2011, A\&A, 527, A36

Martín, S., Mauersberger, R., Martín-Pintado, J., Henkel, C., \& García-Burillo, S. 2006, ApJS, 164,450

Mauersberger, R., Henkel, C., \& Sage, L. J. 1990, A\&A, 236, 63

Meier, D. S., \& Turner, J. L. 2005, ApJ, 618, 259

Meijerink, R., \& Spaans, M. 2005, A\&A, 436, 397

Meijerink, R., Spaans, M., \& Israel, F. P. 2007, A\&A, 461, 793

NED. 2009, NASA Extragalactic Database

Negishi, T., Onaka, T., Chan, K., \& Roellig, T. L. 2001, A\&A, 375, 566

Nguyen-Q-Rieu, Nakai, N., \& Jackson, J. M. 1989, A\&A, 220, 57

Nguyen-Rieu, Viallefond, F., Combes, F., et al. 1994, in Astronomy with Millimeter and Submillimeter Wave Interferometry, ed. M. Ishiguro \& J. Welch, ASP Conf. Ser., 59, IAU Colloq. 140, 336

Papadopoulos, P. P. 2007, ApJ, 656, 792

Pérez-Beaupuits, J. P., Aalto, S., \& Gerebro, H. 2007, A\&A, 476, 177

Prasad, S. S., \& Huntress, Jr., W. T. 1980, ApJ, 239, 151

Regan, M. W., Sheth, K., \& Vogel, S. N. 1999, ApJ, 526, 97

Rodriguez-Franco, A., Martin-Pintado, J., \& Fuente, A. 1998, A\&A, 329, 1097

Sakai, N., Sakai, T., Hirota, T., \& Yamamoto, S. 2008, ApJ, 672, 371

Sakai, N., Sakai, T., Hirota, T., Burton, M., \& Yamamoto, S. 2009, ApJ, 697, 769

Sanders, D. B., Mazzarella, J. M., Kim, D.-C., Surace, J. A., \& Soifer, B. T. 2003, AJ, 126, 1607

Schilke, P., Walmsley, C. M., Pineau Des Forets, G., et al. 1992, A\&A, 256, 595

Solomon, P. M., Downes, D., Radford, S. J. E., \& Barrett, J. W. 1997, ApJ, 478, 144

Sorai, K., Nakai, N., Kuno, N., \& Nishiyama, K. 2002, PASJ, 54, 179

Spoon, H. W. W., Keane, J. V., Tielens, A. G. G. M., et al. 2002, A\&A, 385, 1022

Spoon, H. W. W., Marshall, J. A., Houck, J. R., et al. 2007, ApJ, 654, L49

Stacey, G. J., Geis, N., Genzel, R., et al. 1991, ApJ, 373, 423

Szczepanski, J., Wang, H., Doughty, B., Cole, J., \& Vala, M. 2005, ApJ, 626, L69

Tielens, A. G. G. M., \& Hollenbach, D. 1985, ApJ, 291, 722

Wang, M., Henkel, C., Chin, Y.-N., et al. 2004, A\&A, 422, 883 


\section{Appendix A: Line ratios and source sizes}

We have calculated the line ratios between the observed $\mathrm{HC}_{3} \mathrm{~N}$, $\mathrm{HCN}$, and HNC lines, and the used method will be demonstrated in this appendix. The method accounts for beam size/source size effects, using the following formula for a line ratio between the spectral lines A and B:

$$
\frac{I(\mathrm{~A})}{I(\mathrm{~B})}=\frac{\theta_{\mathrm{mb}_{\mathrm{A}}^{2}}^{2}+\theta_{s}^{2}}{\theta_{\mathrm{mb}_{\mathrm{B}}^{2}}^{2}+\theta_{\mathrm{s}}^{2}} \frac{\eta_{\mathrm{mbB}}}{\eta_{\mathrm{mbA}}} \frac{\int T_{\mathrm{AA}}^{*} \mathrm{~d} v}{\int T_{\mathrm{AB}}^{*} \mathrm{~d} v},
$$

where $\theta_{\mathrm{mb}}$ is the main beam half-power beam width (or main beam size) of the telescope, $\eta_{\mathrm{mb}}$ is the main beam efficiency of the telescope, $\theta_{\mathrm{s}}$ is the source size of the observed object, and $\int T_{\mathrm{A}}^{*} \mathrm{~d} v$ is the integrated intensity of the signal. The temperature can also be given in $T_{\mathrm{mb}}$ scale - in this case, the main beam efficiency corresponding to that observation should be omitted since $T_{\mathrm{mb}}=\frac{T_{\mathrm{A}}^{*}}{\eta_{\mathrm{mb}}}$.

Some of the galaxies have very large angular distributions. The measured intensities might in these few cases not represent a global value for molecular gas in the galaxy, but rather a value for a certain (central) region of the galaxy. This will be discussed in Sect. A.1. If the different observations for the same galaxy are made in different parts of the galaxies the line ratios might be misleading. The exact positions of observation are given in most of the references, and these have been compared. The largest difference in position between two observations in the same galaxy is for IC 342, which has a $3^{\prime \prime}$ difference between the HCN observation in Sorai et al. (2002) and the $\mathrm{HNC}$ and $\mathrm{HC}_{3} \mathrm{~N}$ observations in Henkel et al. (1988). Compared to the sizes of HNC and $\mathrm{HC}_{3} \mathrm{~N}$ distribution (Meier \& Turner 2005) as well as the used beam sizes $\left(19^{\prime \prime}\right.$ and $\left.25^{\prime \prime}\right)$, this offset is although rather small. For all other galaxies, the position difference is at most $1^{\prime \prime}$.

Another issue is that two molecules compared in a line ratio might not have the same spatial distributions, and too narrow a beam might exclude more flux from one molecule than from the other. The problem gets even greater if the two molecular lines are observed with two different beam sizes. The problem mainly affect the most nearby galaxies in the sample, whose source sizes are comparable with the used beam sizes.

The size of the dense molecular region is also important when converting from measured temperature to brightness temperature, and as a consequence of this also when computing the line ratios between two transitions in a galaxy when different telescopes have been used. When available, the source size has been estimated from tables or maps showing the HCN source size, thus assuming that the HCN source size is similar to the source sizes of the other molecules used in the line ratio calculations ( $\mathrm{HNC}$ and $\mathrm{HC}_{3} \mathrm{~N}$ ) as all these lines are expected to be present only in the dense molecular regions (Meier \& Turner 2005). When an HCN source size has not been found, the source size of the $\mathrm{CO}$ emission has been used instead. Preference has then been given to the higher $\mathrm{CO}$ transitions, as these are better tracers of dense gas than CO 1-0, which traces much thinner molecular gas, giving too high a value on the source size. The source sizes used in this work are shown in Table 3, and unless no transition is stated, HCN 1-0 should be assumed.

For some of the galaxies no reliable value on the source size (neither $\mathrm{HCN}$ nor CO) has been found. This might still not be a problem when calculating the line ratios. In Sect. A. 2 it will be shown that the error when assuming a point source is less than $5 \%$ for objects more distant than $45 \mathrm{Mpc}$, and less than $10 \%$ for objects more distant than $30 \mathrm{Mpc}$. These errors are calculated for a line ratio made with the most different beam sizes used in this work. When the beam sizes of the telescopes used for the observations are (almost) the same, the source sizes will just cancel. In any case, this error should be smaller than errors introduced by the use of so many different telescopes and instruments.

\section{A.1. Nearby galaxies}

As mentioned above, some of the most nearby galaxies have source sizes larger than the beam size of the telescope used for the observation. When calculating molecular line ratios, this might pose a problem. Only if we expect the distributions of the two molecules to have the same shape and size, and the observations are made with the same beam sizes, we will achieve the same ratio as for a global measurement on the galaxy. In particular, we expect the $\mathrm{HC}_{3} \mathrm{~N}$ to be concentrated in a smaller region of the galaxy than HCN and HNC (Meier \& Turner 2005), which makes the calculations of these ratios depend on the beam size to be large enough to cover the whole dense molecular region of the galaxy (e.g. the whole $\mathrm{HCN}$ region).

If the beam size is smaller than the $\mathrm{HCN}$ (or $\mathrm{HNC}$ ) emitting region, but larger than the $\mathrm{HC}_{3} \mathrm{~N}$ region, the $\mathrm{HC}_{3} \mathrm{~N} / \mathrm{HCN}$ (or $\mathrm{HC}_{3} \mathrm{~N} / \mathrm{HNC}$ ) ratio will be overestimated, as all $\mathrm{HC}_{3} \mathrm{~N}$ will be seen, but not all $\mathrm{HCN}$ (or HNC). However, if the opposite would be true, the $\mathrm{HC}_{3} \mathrm{~N} / \mathrm{HCN}$ and $\mathrm{HC}_{3} \mathrm{~N} / \mathrm{HNC}$ instead would be underestimated. But since $\mathrm{HCN}$ and $\mathrm{HNC}$ are more abundant than $\mathrm{HC}_{3} \mathrm{~N}$ in all studied sources, this seems very unlikely.

Not knowing the $\mathrm{HC}_{3} \mathrm{~N}$ source size will also affect the line ratios from the more distant galaxies to some extent, since the source size used for the line ratio calculations is an $\mathrm{HCN}$ source size (in a few cases even a $\mathrm{CO}$ source size) also for the $\mathrm{HC}_{3} \mathrm{~N}$ intensities. However, assuming the proportion between the HCN and $\mathrm{HC}_{3} \mathrm{~N}$ source sizes to be similar for all galaxies, this will affect all line ratios in the same way, thus making all line ratios a little bit too high.

Another problem for the line ratios of the nearby galaxies is that the two different observations used to calculate a line ratio sometimes are made with different beam sizes. When comparing $\mathrm{HCN} 1-0, \mathrm{HNC} 1-0$, and $\mathrm{HC}_{3} \mathrm{~N} 10-9$ observations made with the same telescopes the difference in source size is negligible, but if a line ratio is calculated from observations from two different telescopes the two molecular intensities are observed towards regions with different sizes. For distant galaxies this is not a problem, since the whole molecular region of the galaxy is unresolved in any beam. For the more nearby galaxies, one of the molecules might be observed more or less globally in the galaxy, while the other is observed very locally in the galactic centre, giving an erroneous line ratio. The galaxies affected by this issue should be the same as those affected by the previously mentioned beam size issue.

\section{A.2. Unknown source sizes}

The source sizes used in this study are found in Table 3. However, for some sources no reliable value for the source size has been found. We will here discuss why this is not always a problem, and estimate sizes of the errors inflicted from not knowing the source size.

As can be seen above, the line ratios depend on the source and beam sizes with the factor $\frac{\theta_{\mathrm{mb}_{\mathrm{A}}^{2}+\theta_{\mathrm{s}}^{2}}}{\theta_{\mathrm{mb}_{\mathrm{A}}^{2}}+\theta_{\mathrm{s}}^{2}}$. The source size $\theta_{\mathrm{s}}$ can be ignored if both beam sizes $\theta_{\mathrm{mbA}}, \theta_{\mathrm{mbB}} \gg \theta_{\mathrm{s}}$. If the beam sizes $\theta_{\mathrm{mbA}} \approx \theta_{\mathrm{mbB}}$, the source size will also cancel. However, if $\theta_{\mathrm{s}}$ is comparable to the beam sizes, and $\theta_{\mathrm{mbA}}$ and $\theta_{\mathrm{mbB}}$ are nonsimilar, the source size becomes an important factor. 
The error when assuming the source to be point-like, e.g. setting $\theta_{\mathrm{s}}=0$, will be

$$
\frac{\theta_{\mathrm{mb}_{\mathrm{A}}^{2}}^{2} / \theta_{\mathrm{mb}}^{2}}{\frac{\theta_{\mathrm{mb}}^{2}+\theta_{\mathrm{s}}^{2}}{\theta_{\mathrm{mb}_{\mathrm{B}}^{2}}^{2}+\theta_{\mathrm{s}}^{2}}} \text {. }
$$

For the sources where an HCN source size has been used, the corresponding source diameter has never exceeded $1.6 \mathrm{kpc}$ (NGC 2146). For the CO source sizes, the largest is found in NGC 5135, with a source diameter of $4.1 \mathrm{kpc}$ in CO 1-0, but the HCN source sizes should be smaller than this.
Assuming no larger $\mathrm{HCN}$ source diameter than $1.6 \mathrm{kpc}$, and the largest and smallest beam sizes at $90 \mathrm{GHz}$ (SEST with 57" and IRAM with $28^{\prime \prime}$ ), the error will be less than $5 \%$ for distances greater than $45 \mathrm{Mpc}$, and less than $10 \%$ for distances greater than $30 \mathrm{Mpc}$. Thus, even for objects closer than $30 \mathrm{Mpc}$, the error caused by the point-like approximation $\left(\theta_{\mathrm{s}}=0\right)$ is notable only if the $\theta_{\mathrm{mb}}$ of the two observations used to calculate the ratio have notably different sizes (at least a 30\% difference in beam sizes is needed to produce an error of $10 \%$ ). 